\title{
Molecular Gas Kinematics in Barred Spiral Galaxies
}

\author{
Carnegie Institution of Washingtol W, Department of Terrestrial Magnetism, \\ 5241 Broad Branch Road, Washington, DC 20015 \\ Kartik Sheth ${ }^{3}$, \& Stuart N. Vogel ${ }^{4}$ \\ Department of Astronomy, \\ University of Maryland, \\ College Park, MD 20742
}

\begin{abstract}
To quantify the effect that bar driven mass inflow can have on the evolution of a galaxy requires an understanding of the dynamics of the inflowing gas. In this paper we study the kinematics of the dense molecular gas in a set of seven barred spiral galaxies to determine which dynamical effects dominate. The kinematics are derived from observations of the $\mathrm{CO} J=(1-0)$ line made with the Berkeley-Illinois-Maryland Association (BIMA) millimeter array. We compare the observed kinematics to those predicted by ideal gas hydrodynamic and ballistic cloud-based models of gas flow in a barred potential. The hydrodynamic model is in good qualitative agreement with both the current observations of the dense gas and previous observations of the kinematics of the ionized gas. The observed kinematics indicate that the gas abruptly changes direction upon entering the dust lanes to flow directly down the dust lanes along the leading edge of the bar until the dust lanes approach the nuclear ring. Near the location where the dust lanes intersect the nuclear ring, we see two velocity components: a low velocity component, corresponding to gas on circular orbits, and a higher velocity component, which can be attributed to the fraction of gas flowing down the bar dust lane which sprays past the contact point toward the other half of the bar. The ballistic cloud-based model of the ISM is not consistent with the observed kinematics. The kinematics in the dust lanes require large
\end{abstract}

\footnotetext{
${ }^{1}$ Email-mregan@dtm.ciw.edu

${ }^{2}$ Hubble Fellow

${ }^{3}$ Email-kartik@astro.umd.edu

${ }^{4}$ Email-vogel@astro.umd.edu
} 
velocity gradients which cannot be reproduced by an ISM composed of ballistic clouds with long mean-free-paths. Therefore, even the dense ISM responds to hydrodynamic forces.

Subject headings: galaxies: ISM — galaxies: kinematics and dynamics — galaxies: spiral -

\section{Introduction}

Mass inflow in spiral galaxies due to stellar bars has been used to explain a variety of phenomena in galaxy evolution. These phenomena include fueling starbursts (Heller \& Shlosman 1994) and active galactic nuclei (Shlosman, Frank, \& Begelman 1989) and even creating bulges in late-type spirals (Norman, Sellwood, \& Hasan 1996) which may change their Hubble type to an earlier type and eventually destroy the bar (Friedli \& Benz 1993; Norman et al. 1996). Even so, direct observational evidence for mass inflow is sparse. The fundamental problems are that the gas flow is not axisymmetric and only the line-of-sight velocity component is measured. As a result, to date evidence for mass inflow requires comparing observations to models to recover the missing velocity component. This was done for the barred galaxy NGC 1530, where a good match was found between an ideal gas hydrodynamic model (Piner, Stone, \& Teuben 1995; hereafter PST95) and the H $\alpha$ velocity field (Regan, Vogel, \& Teuben 1997; hereafter RVT97). However, H $\alpha$ emission traces relatively diffuse interstellar gas. It would be more convincing to show that dense molecular gas, which probably constitutes the bulk of the gas mass and which may reside in ballistic clouds that do not respond to hydrodynamic forces, also exhibits the kinematics predicted by hydrodynamic models. By observing a sample of galaxies at a variety of viewing angles it should be possible to infer, on average, the missing velocity component and to test whether the hydrodynamic model or ballistic cloud model can best reproduce the kinematics of the dense gas.

In this paper we present $\mathrm{CO} \mathrm{J}=(1-0)$ observations of seven barred spiral galaxies. The galaxies were selected based on their proximity, previous $\mathrm{CO}$ detections, and strength of dust lanes in the Hubble atlas (Sandage 1961). Global characteristics of these galaxies are listed in Table 1. Using this sample, we investigate the kinematics of dense gas in bar dust lanes and in the crucial region where the bar dust lanes approach the nuclear ring and compare the observed gas kinematics to those predicted by hydrodynamic and cloud-based models. 


\section{Observations and Data Reduction}

We observed the $\mathrm{CO} \mathrm{J}=(1-0)$ line toward seven barred spiral galaxies using the Berkeley-Illinois-Maryland-Association (BIMA) Array during the period 1993 to 1997. The details of the observing are listed in Table 2. For all the observations the instrumental gain and phase were calibrated from observations of a nearby quasar approximately every 30 minutes, and structure in the IF passband was calibrated with observations of a strong quasar. The flux density scale was established by using observations of either Mars or Uranus. We estimate the uncertainty in the flux calibration to be $30 \%$.

We formed naturally weighted maps of the $\mathrm{CO}$ data with $10 \mathrm{~km} \mathrm{~s}^{-1}$ channels and cleaned the map using the Högbom algorithm. We then performed an iterative phase-only multiple channel self-calibration process as explained in Regan, Vogel, \& Teuben (1995). The resulting phase corrections were not large $\left(<\sim 30^{\circ}\right)$. For each of the galaxies we determined the noise level, $\sigma$, from emission free regions of the channel maps and formed clipped red and blue total intensity maps by summing all the flux with $\left|S_{\nu}\right|>2 \sigma$ on each side of the systemic velocity (Figs 1-7a) within the velocity limits for each galaxy shown in Table 2. We also formed a total intensity map from the channel maps for each galaxy by summing all the flux over the imaged velocity range in Table 2 (Figs 1-7 b). Fits versions of the data cubes for each galaxy have been placed in the Astronomy Digitial Image Library.

NGC 3627 was reduced differently since it was observed as a mosaic of five pointings. In this case we formed a linear dirty mosaic of the five pointings. We then deconvolved the map using a maximum entropy method (Sault, Staveley-Smith, \& Brouw 1996). We used the resulting model for an iterative phase-only self-calibration. We then primary beam corrected the map and formed the total intensity map. This map is different from the other maps in that the noise level varies over the mapped region.

\section{Results}

\subsection{CO Morphology}

\subsection{1. $N G C 1300$}

NGC 1300 is a prototype strongly barred galaxy (Sandage 1961). Its HI velocity field has been studied by England (1989) and Lindblad et al. (1997). Optical images of the galaxy reveal straight dust lanes along the leading edge of the bar and regions of active star formation at the bar ends. Optical surface photometry reveals an outer star forming ring 
that coincides with the positions of large HI concentrations (Elmegreen et al. 1996).

Due to the low declination of the galaxy, the resulting synthesized beam is elongated north-south and the sensitivity is relatively low. Figure 1a shows that the $\mathrm{CO}$ emission is characterized by two peaks, a strong one west of the nucleus at location $\mathrm{P}$ and a broader weaker one east of the nucleus at location $\mathrm{F}$. There is a weak extension of the $\mathrm{CO}$ extending out to the east from the east peak toward location D.

\subsection{2. $N G C 1530$}

NGC 1530 is one of the closest strongly barred northern hemisphere galaxies. Molecular emission from NGC 1530 has been discussed by Regan et al. (1995), Downes et al. (1996), Reynaud \& Downes (1997), and Reynaud \& Downes (1998). In addition, the mass inflow rate into the central region has been estimated to be $1 \mathrm{M}_{\odot} \mathrm{yr}^{-1}$ from a comparison of $\mathrm{H} \alpha$ Fabry-Perot observations and a hydrodynamic model (RVT97).

Figure 2b shows that the morphology can be characterized by two peaks of emission to the northeast (location F) and southwest (location S) of the nucleus of the galaxy. The emission at each peak is extended along the dust lanes. Higher resolution maps (Regan et al. 1995; Reynaud \& Downes 1997) reveal that there may be an inner spiral or a broken ring of emission interior to the two main emission peaks.

\subsection{3. $N G C 2903$}

NGC 2903 is a starburst galaxy that is classified as weakly barred in the optical, although near infrared images (Regan \& Elmegreen 1997) reveal a strong bar. Radio continuum, optical, and near infrared imaging of the nuclear region show a complex structure of "hot spots" of active star formation (Wynn-Williams \& Becklin 1985; Jackson et al. 1991). These hot spots are within the region of strong concentration of molecular gas seen in single dish CO observations (Jackson et al. 1991; Planesas, Colina, \& Perez-Olea 1997).

Figure $3 \mathrm{~b}$ reveals that this galaxy does not show two peaks of emission at the inner ends of the dust lanes. Instead, there is a central peak of emission whose spectrum contains a single broad velocity component centered on the systemic velocity of the galaxy. There is a broad extension of $\mathrm{CO}$ emission along the southern half of the bar and a narrower extension along the northern half of the of the bar. In addition, this galaxy shows a concentration of emission at each end of the bar. 


\subsection{4. $N G C 3627$}

NGC 3627 is member of the Leo triplet; NGC 3623 and the edge-on NGC 3628 make up the other two-thirds. The triplet shows strong evidence of interaction as indicated by an HI tail 80 kpc east of NGC 3628 (Rots 1978; Haynes, Giovanelli, \& Roberts 1979). There is high star formation activity in both NGC 3627 and NGC 3628 based on radio continuum observations (Reuter et al. 1991) and high IRAS flux densities (Rice et al. 1988; Young et al. 1989). NGC 3627 is the most prominent member of the group with a pronounced dust pattern (Sandage 1961) indicating a strong spiral density pattern. Measurements from the Kuiper Airborne Observatory at 50 and $100 \mu \mathrm{m}$ by Smith et al. (1994) found the far

IR to $\mathrm{H} \alpha$ ratio to be very high, implying large extinction in the central region. NGC 3627 has been previously observed in $\mathrm{CO}(1-0)$ and $\mathrm{CO}(2-1)$ with the IRAM 30m (Reuter et al. 1996) and with the 3-element BIMA interferometer in $\mathrm{CO}(1-0)$ (Zhang, Wright, \& Alexander 1993). Our new 9-element observations have a larger field of view and greater sensitivity than the previous BIMA observations.

The CO map shown in Figure Ga is different than the others in this paper since it was formed from a mosaic of five pointings. This yielded a very large field of view allowing the global CO morphology to be viewed. As previously seen by Zhang et al. (1993), the center of the galaxy shows a single emission peak on the nucleus of the galaxy extending out into two offset ridges. Both ridges connect to the large $\mathrm{CO}$ concentrations at the ends of the bar. The two emission peaks at the bar ends are quite strong and the $\mathrm{CO}$ can be seen to connect to the spiral arms. Here, as in NGC 2903, the CO emission in the central CO peak has only one velocity component centered on the systemic velocity of the galaxy.

\subsection{5. $N G C 4314$}

NGC 4314 is an SBa galaxy with a 140" bar and two faint spiral arms tracing 130 degrees of arc out to a radius of $125^{\prime \prime}$. H $\alpha$ imaging of the galaxy shows it to have a nuclear ring of star formation (Pogge 1989). NGC 4314 has been observed in CO J=(1-0) with the OVRO interferometer (Benedict, Smith, \& Kenney 1996). HST observations reveal a ring of star forming knots coincident with the $\mathrm{H} \alpha$ ring (Benedict et al. 1993).

Figure 5 $5 \mathrm{~b}$ shows that there are two peaks of emission in NGC 4314. They are not located at the inner terminus of the dust lanes since the dust lanes terminate to the northeast and southwest of the nucleus while the two peaks are to the northwest and southeast. Although it is not clear from our map, the higher resolution OVRO maps show that the CO is distributed in a ring (Benedict et al. 1996). 


\subsection{6. $N G C 5135$}

NGC 5135 has been called a mini-Seyfert 2 (Phillips et al. 1983; Thuan 1984). It was detected in the single dish CO survey of Heckman et al. (1989). For our observations the low declination of this galaxy yielded a very elliptical beam. Figure $6 \mathrm{~b}$ shows that the $\mathrm{CO}$ emission is only partially resolved by our beam and is centered on the nucleus of the galaxy with a small extension visible to the southeast along the leading edge of the bar. The spectrum of the emission from the central region shows a single velocity component centered on the systemic velocity of the galaxy similar to what is seen for the central regions of NGC 2903 and NGC 3627.

\subsection{7. $N G C 5383$}

NGC 5383 is a relatively nearby northern hemisphere barred spiral which has been used for many models of gas flow in barred potentials (Sanders \& Tubbs 1980; Duval \& Athanassoula 1983). Optical studies have described the morphology of the galaxy in detail (Burbidge, Burbidge, \& Prenderdast 1962; Duval \& Athanassoula 1983; Elmegreen \& Elmegreen 1985, Barton \& Thompson 1997). The galaxy is inclined at $50^{\circ}$ and has a prominent bar $\left(110^{\prime \prime}\right)$ at a position angle of $135^{\circ}$ (Sheth et al. 1998; Duval \& Athanassoula 1983), classic offset dust lanes and faint spiral arms. Kinematics of the galaxy have been studied using slit spectroscopy (Burbidge et al. 1962; Peterson et al. 1978; Duval \& Athanassoula 1983), HI interferometry (Sancisi, Allen, \& Sullivan 1979). Extensive hydrodynamic modeling of the galaxy has been done by Huntley (1978) and Athanassoula (1992b). Orbital dynamics have been studied by Sanders \& Tubbs (1980), Tubbs (1982), and Athanassoula (1992a). This galaxy is the subject of an extensive multi-wavelength study in Sheth et al. (1998).

Figure 7b shows that a large fraction of the detected CO emission from NGC 5383 is in two peaks at the inner ends of the dust lanes. In addition, it also shows an extended region of emission between the peaks of emission and a weaker peak centered on the nucleus. There is also faint emission extending from the two strong peaks along the dust lanes.

\subsubsection{CO Morphology Summary}

Three of the seven galaxies (NGC 1300, NGC 1530, and NGC 5383) exhibit the "twin peaks" morphology described by Kenney et al. (1992). This morphology is characterized by two emission maxima near the nucleus, generally at the inner terminus of the straight 
offset bar dust lanes. Three of the other four galaxies (NGC 2903, NGC 3627, and NGC 5135) have essentially unresolved emission centered on the nucleus. The spectra of this emission show a single broad velocity component. Only NGC 4314 shows a ring of molecular emission.

Another region in barred galaxies where we would expect to detect emission is from the ends of the bars (Handa et al. 1990, Kenney \& Lord 1991, Downes et al. 1996). The fact that we only detect gas at the ends of the bar in two of the seven galaxies (NGC 2903 and NGC 3627) observed in this paper is not significant. In all galaxies but NGC 3627 we observed at only a single position causing the flux from the bar ends to be severely attenuated by the primary beam of the antenna. Of the single pointing galaxies, only in NGC 2903 was the bar end both close enough to the center of the field and strong enough to detect. Since single dish CO observations of NGC 1530 revealed concentrations of gas at the ends of the bar (Downes et al. 1996), we know that such concentrations can exist and not be detected in our maps.

\section{CO Kinematics}

RVT97 showed that the H $\alpha$ velocity field of NGC 1530 is in good agreement with the global velocity field of a barred galaxy predicted by hydrodynamic models (PST95). Both the model and observations showed a strong shock along the entire leading edge of the bar, isovelocity contours which become parallel in the nuclear region, a $10^{\circ}$ twist in the inner kinematic major axis, and kinks in the isovelocity contours in the spiral arms. There are several potential problems with using $\mathrm{H} \alpha$ as a tracer of the ISM kinematics: $\mathrm{H} \alpha$ emission arises from the diffuse gas in the ISM, can be biased by regions of active star formation, and is subject to extinction by dust. We would like a tracer of the gas that does not suffer these biases and one that traces the dense molecular gas that probably constitutes the bulk of the gas mass. Carbon monoxide is just such a tracer, allowing it to provide independent confirmation of the model. Because CO emission only arises from regions where the ISM is molecular, we can determine what we would expect to observe for the CO kinematics by looking at the kinematics of the dense gas in the model.

We have chosen to focus on comparing the kinematics to the models rather than the morphology because the models that we are using do not attempt to take into consideration any consumption of gas by star formation. Since the removal of gas over time can be quite large, we would not expect the morphology of the models and observations to match. On the other hand, the kinematics should not be changed by the removal of gas by star formation and so are a better test of the models. 


\subsection{Hydrodynamic Model Predictions}

Figure 8 is a plot of the gas surface density from the high resolution hydrodynamic model (run 4) of PST95 near the end of the simulation after it has reached a nearly steady state configuration. Three different regions are labeled in the figure: the leading dust lane, the contact point where the dust lane meets the nuclear ring, and a spray region downstream of the contact point and the nuclear ring where the gas flow is divergent.

Figure 9 shows the model gas velocity field in the plane of the galaxy in a reference frame rotating with the bar. From the figure we can see that the high density gas in the dust lane is moving directly down the dust lane. At the contact point the gas in the nuclear ring is moving on nearly circular orbits, while the gas just outside of the ring is moving faster than the gas in the ring. Finally, in the spray region the gas that is spraying back out into the bar is moving faster than the gas in the nuclear ring. Here gas is in a divergent flow as it leaves the contact point.

Since we do not observe the velocity in the plane of the galaxy but only the line-of-sight velocity, the observed velocity of the gas will be affected by the orientation of the galaxy on the sky. Along the galaxy major axis we only observe velocity that is tangential in the plane of the galaxy. While on the galaxy minor axis, we only observe motion that is radial in the plane of the galaxy. Moreover, the sign of the projected galactic radial motion depends on whether the observed location is on the near or the far side of the galaxy. For example, on the far side, radial inflow is observed blue-shifted relative to the systemic velocity, while on the near side it is red-shifted. Therefore, interpretation of the kinematics requires knowledge of both the galaxy position and which side of the galaxy is the near side. For all of the galaxies in this paper, we use the usual assumption that the spiral arms are trailing to allow us to determine the near side of the galaxy and the convention that negative radial velocities in the plane of the galaxy are inward.

Consider first gas in the bar dust lane located far from the nuclear ring. Figure 9 shows that in the bar frame such gas has very little tangential motion but a large radial inward velocity. The additional tangential motion resulting from the bar rotation (this ranges from $20 \mathrm{~km} \mathrm{~s}^{-1} \mathrm{kpc}^{-1}$ to $60 \mathrm{~km} \mathrm{~s}^{-1} \mathrm{kpc}^{-1}$ ( Teuben et al. 1986; Buta 1986; Jorsater \& van Moorsel 1995; England, Gottesman, \& Hunter 1990; Merrifield \& Kuijken 1995; Ryder, Buta, \& Toledo 1996; Buta \& Purcell 1998)) can generally be estimated. However, we note that if the bar dust lane lies close to the minor axis then there is no Doppler contribution from bar rotation, and motion along the dust lane is directly observed. On the other hand, if the observed location is close to the major axis, then the observed velocity depends little on the flow speed along the dust lane, but rather on the pattern speed of the bar. 
Consider second gas in the bar dust lane located near the nuclear ring (i.e. near the contact point). Figure 9 shows that in this case gas is flowing nearly tangentially. Due to beam-smearing, emission observed at this location comes not only from this dust lane gas, but also from gas rotating in the nuclear ring. The latter gas is also moving tangentially but at a lower speed than the dust lane gas. Therefore, near the contact point we expect spectra to show two components, corresponding to the dust lane gas and the nuclear ring gas. The nuclear ring gas has a lower tangential speed and will therefore be the component closer to the systemic velocity of the galaxy. In some cases, the velocity separation between the two components will be too small to resolve the two components.

Finally, consider gas in the spray region (i.e. downstream from the contact point). This gas has both an outward radial velocity and a tangential velocity that is greater than or equal to the gas in the nuclear ring. Again given the finite resolution of the CO observations we would expect that the spraying gas spectra will be superimposed upon the spectra of the denser gas in the nuclear ring. The relative velocities of these two components will depend upon the projections in the specific galaxy.

\subsection{Cloud-based Model Predictions}

An alternative to the hydrodynamic approach to modeling the ISM is to assume that the ISM is composed of an ensemble of colliding gas clouds that move on ballistic orbits (Schwarz 1984, Roberts \& Hausman 1984, Combes \& Gerin 1985, Palous, Jungwiert, \& Kopecky 1993, Byrd et al. 1994). In this approach the ISM does not experience any pressure forces and thus there are no large-scale shocks. Due to the collisions in the cloud ensemble, the cloud orbits will lead the bar within the bar radius (Combes 1996), resulting in orbits such as those shown in Figure 10.

From these gas orbits we can make predictions about what the observed kinematics should be if the ISM is well approximated by an ensemble of gas clouds. First, consider the gas clouds in the bar dust lanes far from the nuclear ring. Figure 10 shows that the dust lane is formed from the crowding and superposition of several orbits with small differences in total energies. All of these velocities will have the same direction since the various orbits are nearly parallel in the dust lanes. The tangent vector to the orbits in the dust lane shows that the clouds in the dust lanes are not moving as directly inward as the gas in the dust lanes shown in Figure 9. This $30-40^{\circ}$ difference in the direction of the gas flow would be difficult to detect unambiguously. The biggest difference in the predicted kinematics in the dust lane comes in the gas that is upstream of the dust lane. In the cloud-based model there is not a shock or a large change in gas velocity in the dust lane. The gas upstream of 
the dust lane has almost the same speed and direction of flow as the gas in the dust lane. It is flowing inwards with low but non-zero tangential velocity. This is in contrast to the gas upstream of the dust lane in the hydrodynamic model where the gas has a much different direction of flow from the gas in the dust lanes.

While the contact point is not well defined in the cloud-based model, the expected kinematics at the observed contact point are similar to those in the hydrodynamic model. We would expect to see slow moving gas in the nuclear ring superimposed on the faster moving clouds that are moving past the ring.

Similarly, in the spray region Figure 10 shows that the gas orbits are diverging and heading around to contact the dust lane on the other side of the bar. Although the gas orbits are not diverging from a single point as the gas streamlines do in the hydrodynamic model, the differences are very small. Therefore, the expected kinematics are the same for the two models of the ISM in this region.

\subsection{Observed Kinematics}

To investigate the gas kinematics we have chosen to focus on the gas as it flows down the dust lane. The dust lanes and nuclear region are where the ISM density is high enough to detect molecular gas. The dust lanes are also the critical region for the predictions of the hydrodynamic model since the dust lanes are where the cloud-based and hydrodynamic models predict different kinematics. For each dust lane of each galaxy we extracted a series of spectra along the leading edge of the bar extending to the contact point and continuing out into the spray region. These locations may not exactly match the locations of the observed dust lanes but allow us to use a consistent set of locations for all the galaxies. The differences between where the spectra are extracted and the actual locations of the dust lanes are small relative to the sizes of the synthesized beams. Each spectrum is separated by approximately the size of the synthesized beam. The galaxy position angles used for this analysis are shown in Table 3. The locations of each spectrum, the galaxy axes, approaching and receding sides, and near and far sides are shown in Figures 1-7b.

\subsubsection{NGC 1300}

These spectra have a low signal to noise ratio which when combined with the large elliptical synthesized beam leads to spectra that are difficult to interpret. Even so, at several locations we detect kinematic features that are significant. At location $\mathrm{P}$ we see 
gas that is close to the major axis of the galaxy and in a dust lane. The spectrum at this location shows two velocity components. One is about $70 \mathrm{~km} \mathrm{~s}^{-1}$ wide centered on the systemic velocity and the other is red-shifted relative to the systemic velocity of the galaxy. The component centered on the systemic velocity must have very little tangential velocity; this is consistent with the inflowing gas in the dust lanes. The other component seen at this location must have significant tangential velocity; this is consistent with this being gas in near-circular rotation at the nuclear ring. Presumably the two components are spatially distinct but are not resolved by our beam.

The spectrum at location $\mathrm{H}$, which is near the spray region of the eastern dust lane and the nuclear ring, shows weak broad blue-shifted emission with velocities of $100 \mathrm{~km}$ $\mathrm{s}^{-1}$ relative to the systemic velocity. Since this location is approximately on the near side minor axis, this gas may be flowing radially outward in the plane of the galaxy or it may be that emission here is beam-smeared from the other side of the galaxy. There is also some gas red-shifted as much as $50 \mathrm{~km} \mathrm{~s}^{-1}$ relative to systemic velocity but this is clearly due to emission beam-smeared from the the strong emission seen at location P. The relatively large synthesized beam means that an unambiguous interpretation of the spectrum at this location is not possible.

\subsection{2. $N G C 1530$}

In the eastern dust lane of NGC 1530 we first detect faint emission at location A. There is weak emission near systemic and at $2580 \mathrm{~km} \mathrm{~s}^{-1}$ which is about $120 \mathrm{~km} \mathrm{~s}^{-1}$ red-ward of systemic. At this location between the receding major axis and the far side minor axis, only if the gas has a significant radial inward velocity will it have an observed velocity near systemic.

Moving in along the eastern dust lane of NGC 1530 we detect emission at location C. Here the major component is slightly offset to the blue from the systemic velocity. This position in NGC 1530 is about $30^{\circ}$ from the far side minor axis on the receding side of the galaxy. Therefore, gas in circular orbits will be red-shifted relative to the systemic velocity. For gas to be at the observed blue-shifted velocity it would have to have some combination of slower than circular tangential velocity and inward radial motion. Since this is in the dust lane, that is exactly the effect we expect to see. In addition, a weak narrow detection of gas at near circular velocities is seen at $2570 \mathrm{~km} \mathrm{~s}^{-1}$. The observed spread in velocities at this location $\left(150 \mathrm{~km} \mathrm{~s}^{-1}\right)$ would be hard to explain if the ISM were composed of ballistic clouds. 
Location $\mathrm{E}$ is very close to the far side minor axis of the galaxy. Here we see very broad emission from $2320 \mathrm{~km} \mathrm{~s}^{-1}$ to $2520 \mathrm{~km} \mathrm{~s}^{-1}$. Since this location is on the far side of the galaxy inflowing gas will be blue-shifted and outflowing gas will be red-shifted. The observations show that the majority of the gas is inflowing. The inflowing gas is consistent with our expectation of what gas should be doing in the dust lane. The small amount of red-shifted emission that we see is due to beam smearing of gas from the receding side of the galaxy

Location $\mathrm{F}$ is at the contact point of the dust lane and the nuclear ring. This location is also close to the major axis. The spectrum shows that all of the gas is blue-shifted with the strongest component centered on $2350 \mathrm{~km} \mathrm{~s}^{-1}$. A second possible component, at a more blue-shifted velocity than the majority of the gas, is seen at a velocity of around $2280 \mathrm{~km}$ $\mathrm{s}^{-1}$. This second component is what would be expected for the higher velocity gas that is streaming past the nuclear ring.

The existence of this higher velocity component is confirmed by the spectrum at location $\mathrm{H}$. This spectrum is on the near side of the galaxy close to the major axis. There is clearly gas at velocities from $2260 \mathrm{~km} \mathrm{~s}^{-1}$ to $2320 \mathrm{~km} \mathrm{~s}^{-1}$. This is gas in the spray where we expect the gas to have a high tangential velocity and be moving outward from the center of the galaxy. Given the projection of NGC 1530, these two effects will add constructively so we see this as a blue-shifted velocity component relative to the gas on circular orbits in the nuclear ring.

In the western dust lane of NGC 1530 the first significant emission that is detected is at location M. Here we detect broad emission extending from systemic to $100 \mathrm{~km} \mathrm{~s}^{-1}$ blue-ward. Emission over such a large range in velocity is not consistent with the ISM in the dust lanes being composed of primarily dense clouds.

Moving inward toward the nucleus significant emission is seen at location $\mathrm{R}$. This location is the mirror image of location $\mathrm{E}$ in that it is on the near side minor axis. The spectrum looks like the reflection of the spectrum at location $\mathrm{E}$ as well. The majority of the emission is red-shifted relative to the systemic velocity with emission detected out to 2640 $\mathrm{km} \mathrm{s}^{-1}$ approximately $170 \mathrm{~km} \mathrm{~s}^{-1}$ from systemic. This red-shifted gas is flowing inward in the plane of the galaxy. The small amount of emission that is seen blue-ward of systemic velocity is probably due to beam smearing of gas from the ring region.

Location $\mathrm{S}$ is at the contact point of the dust lane and the nuclear ring. This location is about midway between the near side minor axis and the red major axis. We expect gas to be moving completely tangentially here, and the large amount of gas that is red-shifted relative to systemic is consistent with that. We do not detect the expected higher velocity 
component here. The gas that is seen around systemic velocity is unexpected. If this were emission from gas at location $\mathrm{S}$ then the gas would be flowing outward.

At location $\mathrm{T}$ we are slightly downstream of the contact point and on the major axis of the galaxy. Here we see that the red wing of the velocity profile shows a component that is rotating much faster. This is consistent with the fast moving gas that is moving past the nuclear ring.

Finally, at location $\mathrm{U}$, we are well past the contact point and in the spray region. Here the gas is slightly on the far side of the major axis. The high velocity component seen at location $\mathrm{T}$ is not seen here although we do see emission at velocities up to $2610 \mathrm{~km} \mathrm{~s}^{-1}$.

\subsection{3. $N G C 2903$}

Although the emission in NGC 2903 is strong, the central emission is very close to the nucleus of the galaxy and the synthesized beam does not resolve it. This leads to large amounts of beam smearing resulting in large velocity widths.

At location $\mathrm{B}$ on the the north side of the galaxy we do detect gas at the end of the bar. Since in NGC 2903 the bar major axis is closely aligned with the major axis of the galaxy, along the bar we are observing mainly motion that is tangential in the plane of the galaxy. The observed velocity of the gas at location $\mathrm{B}$ is only $60 \mathrm{~km} \mathrm{~s}^{-1}$ from systemic. This observed velocity is small compared to the observed HI line width of $380 \mathrm{~km} \mathrm{~s}^{-1}$ (de Vaucouleurs et al. 1991). Since at this location we are observing gas $2.5 \mathrm{kpc}$ from the nucleus we would expect that the circular velocity would be a larger fraction of the total line width. Location B is on the near side of the galaxy so inflowing gas will be red-shifted. Therefore, this component must have some combination of lower than circular tangential velocity and inward radial velocity. Further inward at location $\mathrm{F}$ in the northern dust lane there is gas at velocities close to the systemic velocity. Since F is close to the near-side minor axis this is what would be expected for gas on circular rotation. At F we also see a component that is blue-shifted implying either fast tangential motion or outward flowing gas. This emission is probably due to beam smearing from either the nuclear region or from the nearby major axis.

Since we do not see the standard twin peak morphology in NGC 2903, the contact point and the spray region are not easy to locate. Location $\mathrm{G}$, which is past the contact point of the northern dust lane, is between the near side minor axis of the galaxy and the receding major axis. We can see that there is gas both blue-ward and red-ward of systemic. The relatively large size of the synthesized beam compared to the galactic radius at this 
location means that the spectrum contains emission from a variety of projections making it impossible to interpret.

In the southern dust lane we also detect gas near the bar end in at location M. This gas, like the gas at location $\mathrm{B}$, is rotating too slowly to be in a circular orbit. In the dust lanes at location $\mathrm{P}$ we detect emission extending over a broad range of velocity $\left(520 \mathrm{~km} \mathrm{~s}^{-1}\right.$

to $700 \mathrm{~km} \mathrm{~s}^{-1}$ ) with a peak near systemic. Emission over such a large range of velocity near the bar dust lanes is not consistent with the expectations of an ISM composed of ballistic clouds. Since location $\mathrm{P}$ is on the far side of the receding major axis, circular motion should be red-shifted. Emission could approach systemic by having a combination of low tangential velocity and inward radial velocity.

This same effect (low tangential velocity and inward radial motion) is seen at $\mathrm{Q}$ where the emission is blue-shifted from systemic. Since $\mathrm{Q}$ is about $60^{\circ}$ from the minor axis and NGC 2903 has an inclination of $60^{\circ}$, the projected radial component is only $43 \%$ of the velocity in the plane of the galaxy. Thus, for gas to be seen $40 \mathrm{~km} \mathrm{~s}^{-1}$ blue-ward of systemic requires the gas to be flowing inward with a velocity of at least $90 \mathrm{~km} \mathrm{~s}^{-1}$. Note that this is a lower limit because any tangential component of velocity will red-shift the emission requiring an even greater inflow speed to yield the same observed velocity.

At location $\mathrm{R}$ there is a broad emission profile extending to both sides of the systemic velocity. As with location $\mathrm{Q}$, in order for there to be emission blue-ward of systemic requires the gas to be flowing radially inward. Gas here is detected $60 \mathrm{~km} \mathrm{~s}^{-1}$ blue-ward of systemic requiring a minimum inflow speed of $130 \mathrm{~km} \mathrm{~s}^{-1}$.

\subsection{4. $\quad N G C 3627$}

Along the southern dust lane of NGC 3627 we see emission all the way from the bar end to the nuclear region. Location $\mathrm{M}$ is near the end of the bar and the spectrum at this location shows a rotation velocity centered around $910 \mathrm{~km} \mathrm{~s}^{-1}$. Location $\mathrm{P}$ is on the major axis of the galaxy and in the dust lane. The spectrum at this location shows an emission peak around $790 \mathrm{~km} \mathrm{~s}^{-1}$ with a large red wing to the emission. Given the higher rotational velocity seen at location $\mathrm{M}$, the slower rotating gas at location $\mathrm{P}$ in the dust lane must have a significantly lower tangential velocity consistent with gas flowing directly down the dust lane.

The fact that there is also gas at location $\mathrm{P}$ with significant tangential velocities implies that we are detecting gas before it enters the dust lane while it still has significant tangential velocities. This large change in velocities at the dust lane is not consistent with 
the expectations of the cloud-based model.

At location $\mathrm{Q}$ we are looking just off the major axis on the far side of the galaxy. Here we see a spectrum similar to the one at location $\mathrm{P}$. Therefore, the bulk of the gas is not rotating fast enough to be in circular orbits.

In the northern dust lane we detect emission near the bar end at location A. Similar to location $\mathrm{M}$ in the southern dust lane, except that the emission is offset to the blue, here we can see that the rotational velocity is centered around $560 \mathrm{~km} \mathrm{~s}^{-1}$. Further down the dust lane at locations $\mathrm{E}$ and $\mathrm{F}$ we can see that at these locations, which are very near the major axis, the gas is rotating much slower than at A. Both locations have emission very near to systemic. Given the minimum tangential velocity provided by the rotation of the bar, the bulk of the gas at location $\mathrm{F}$ must have a very small tangential component of velocity in the rotating reference frame of the bar. Again the large observed line width at location $\mathrm{F}$ is not consistent with the expectations of a ballistic cloud-based ISM.

The nuclear region of NGC 3627 is not resolved by our synthesized beam. Therefore, it is not possible to determine where the contact point of the dust lane and the nuclear ring is. We can see that spectra the include the minor axis (locations $\mathrm{H}$ and $\mathrm{S}$ ) reveal emission from a large range in velocity. This arises from the large beam relative to the galactic radius. This causes the spectrum to include emission from many different projections. much is due to beam smearing. Since we expect the gas in the ring and the gas spraying past the ring to be moving purely tangentially here, observations of the galactic radial velocity should not reveal two components. The galaxy is not favorably oriented for the spectra in the region where there would be a spray (locations $U$ and $J$ ) to show the spray component. This is because the spray should have high tangential velocity and outward radial velocity. The orientation of NGC 3627 is such that at locations $U$ and J these two effects have opposite Doppler effects and thus the spraying gas is hard to differentiate from gas on circular orbits.

\subsection{5. $N G C 4314$}

Our map of NGC 4314 does not detect any gas in the dust lanes. At the location where the dust lane joins the nuclear ring in the north, location D, the spectrum shows a single broad velocity component with velocities on both sides of systemic. Since location D is between the far-side minor axis and the receding side major axis, circular rotating gas will be red-shifted and inflowing gas will be blue-shifted. Therefore, the only way to get gas with velocities blue-ward of systemic is for the gas to be flowing inward. Similarly, at the

other dust lane terminus, location $\mathrm{Q}$, where we are observing gas very near the approaching 
major axis, we see a red wing to the emission profile indicative of gas with a combination of slow tangential velocities and inward radial motion. In a more sensitive OVRO map of NGC 4314, Benedict et al. (1996) also show that at these locations gas is streaming radially inward. In the expected spray region (locations $\mathrm{T}$ and $\mathrm{F}$ ) we do not detect any significant emission.

\subsubsection{NGC 5135}

Interpretation of the kinematics in NGC 5135 is difficult due to the elliptical beam that blends the kinematics. Even so, there are several features that can be unambiguously detected.

At location $\mathrm{D}$ in the dust lane we are looking at gas that is between the near-side minor axis and the approaching major axis. Since this is on the near side of the galaxy, gas that is moving inward radially will have emission red-shifted toward systemic. Because this location is between the major and minor axis we are seeing the blending of radial inflow and tangential rotation. The gas in the dust lane is flowing inward with very little tangential velocity. The radial inflow and low tangential velocity combine to make the gas have more red-shifted emission than gas on circular orbits would have. In fact, that is what we see in the spectrum. There is one spectral feature at a velocity of approximately $4020 \mathrm{~km} \mathrm{~s}^{-1}$ and another stronger feature at a velocity of $4060 \mathrm{~km} \mathrm{~s}^{-1}$. This second feature even has a red wing that extends red-ward of systemic. Gas can be observed with these velocities only if it is moving radially inward.

Location $\mathrm{E}$ is near the contact point of the southern dust lane and again is between the near-side minor axis and the approaching major axis. Here again we are seeing the blending of tangential and radial motions. The velocity profile is asymmetrical with gas in the blue wing having high relative tangential motions. There is also gas red-ward of systemic which could be indicative of inflowing gas. Unfortunately, the large elliptical beam means that the spectrum at location $\mathrm{E}$ is from a very large area including the receding side of the galaxy. Therefore, it is not possible to definitively state that there is inflowing gas at this location.

In the northern dust lane we detect gas at location P. Here we are looking near the receding major axis on the far side of the galaxy. We see gas here with a velocity that is blue by as much as $50 \mathrm{~km} \mathrm{~s}^{-1}$ relative to systemic here. Only by flowing radially inward can gas be seen blue-ward of systemic at this location. Therefore, the gas here must have a low tangential velocity and be streaming radially inward. 


\subsection{7. $N G C 5383$}

In NGC 5383 we do not detect any CO in the dust lanes except near where the dust lanes join the ring. At Location $\mathrm{B}$ on the western side of the galaxy we detect the first significant emission. At this location inflowing gas will be blue-shifted due to its inward radial motion and red-shifted toward systemic due to its low tangential motion. Therefore, these two Doppler effects compete against each other making it hard to determine whether we are seeing inflow.

Location $\mathrm{D}$, at the contact point of the dust lane and the nuclear ring, is almost on the major axis of the galaxy; therefore, we are observing mostly tangential motion in the plane of the galaxy. From the hydrodynamic model we would expect to see the gas in the ring rotating at near circular velocity. This component is seen to peak at a velocity of $2180 \mathrm{~km}$ $\mathrm{s}^{-1}$. A second faster moving component is also seen with its velocity centered at around $2110 \mathrm{~km} \mathrm{~s}^{-1}$. This is, presumably, the gas that is streaming past the ring.

As we follow the dust lane out into the spray region, we can see this higher velocity component remaining. At locations F and G, well into the spray region, we detect gas with a velocity of around $2110 \mathrm{~km} \mathrm{~s}^{-1}$. At this location we are on the near side of the galaxy so the expected radial outflow will be blue-shifted relative to the systemic velocity. The expected higher tangential component will also be blue-shifted so the two Doppler effects combine here allowing a clear detection of the spraying gas. The fact that the faster moving component is stronger relative to the slower moving component is evidence that we are not just seeing emission blending out to this location from the stronger emission at location D. This is because at location $\mathrm{D}$ the slower moving component is much stronger than the faster moving component.

On the eastern side of the galaxy we detect gas in the dust lane as it nears the ring. Here too, the projections of the combination of low tangential motion and radially inflowing gas interfere, making it impossible to determine if the gas motion in the dust lane is directly down the dust lane.

At the contact point of the dust lane and the ring, location $\mathrm{P}$, we do see a red wing to the emission. Since on this side the contact point is also close to the major axis, this gas in the red wing of the profile is gas with a higher tangential velocity. Again we are seeing the gas that is moving past the ring.

As we move out into the spray region we also detect a higher velocity component to the emission. On this side of the galaxy the combination of radial outflow and high tangential velocity combine to red-shift the gas as we see at locations $Q, R$, and weakly at $\mathrm{S}$. This is the gas that is spraying around to contact the western dust lane. 


\section{Discussion}

Overall, the hydrodynamic model is in better agreement with the CO kinematics than the ballistic cloud model. In the dust lanes we detect gas with low tangential velocity in NGC 1300, NGC 1530, and NGC 3627. We detect gas that has some combination of radial inflow and low tangential velocity in NGC 2903, NGC 4314 and NGC 5135. In NGC 5383 the projection of the galaxy precluded us from distinguishing streaming motions from circular motions in the dust lanes. Therefore, the prediction of the hydrodynamic model that the gas in the dust lanes is flowing directly down the dust lanes is consistent with all of our observations.

Recently more sensitive observations of NGC 1530 made by combining observations from the IRAM interferometer with observations from the IRAM 30m confirm our interpretations (Reynaud \& Downes 1998). These observations show that CO is moving 50 to $100 \mathrm{~km} \mathrm{~s}^{-1}$ radially outward upstream of the dust lanes and 70 to $150 \mathrm{~km} \mathrm{~s}^{-1}$ radially inward in the dust lanes.

The expectations of an ISM dominated by ballistic clouds are not consistent with either our observations of NGC 1530, NGC 2903, and NGC 3627 or previous ones of NGC 1530 (RVT97; Reynaud \& Downes 1998). The sharp decreases seen in the tangential velocity at the dust lanes cannot be explained by collisions of clouds with long mean-free-paths. Instead the ISM in this region seems to be well represented by an ideal gas approximation. This does not preclude the molecular gas from being in clouds. It only implies that the clouds must respond to hydrodynamic forces since the velocities of the dense gas change quickly upon entry to the dust lanes.

At the contact point we detect a higher velocity component in NGC 1530 and NGC 5383. These are the only two galaxies in the sample where the contact point is near the major axis of the galaxy. Since the expected higher velocity component is moving completely tangentially in the plane of the galaxy, only when we observe the contact point near the major axis of the galaxy will we be able to detect the higher velocity component. Therefore, given the projections, the expectations of the hydrodynamic model are consistent with our observations.

The gas at the expected velocities of the spray region is found in NGC 1530 and NGC 5383. Since gas in the spray region is flowing outward and has a higher than circular tangential velocity, only at certain orientations will it be distinguishable from the much stronger emission arising from the nuclear region. Only if we observe the spray region in the receding far side or approaching near side quadrants of a galaxy will the projection cause the outward radial and faster tangential Doppler effects to have the same sign. Otherwise, 
the two Doppler effects will have opposite signs and it will not be possible to distinguish spray emission from ring emission. In every galaxy where the projections are favorable we do detect the spray.

The good agreement seen here between the hydrodynamic model and the observations has important implications for modeling of gas flow in barred galaxies. It was possible that the good fit to the hydrodynamic model obtained from the $\mathrm{H} \alpha$ observations of NGC 1530 (RVT97) could have been due to the fact that the $\mathrm{H} \alpha$ was tracing the diffuse ISM while the bulk of the ISM remained in clouds that were not correctly modeled by an ideal gas approximation. Our current CO observations show that the ideal gas model also matches well the kinematics of the dense gas and that the ballistic cloud approximation of the ISM is not correct within the region of the bar.

Higher resolution and more sensitive observations are needed to confirm these suggestions. The projections of the velocities change quickly in the nuclear regions and observations at higher resolution would decrease the beam smearing problem. For example, the synthesized beam for our observations of NGC 5135 has linear dimensions of 3 x $1 \mathrm{kpc}$. This creates a large ambiguity in where the actual location of the emission.

\section{Conclusions}

We have shown that a hydrodynamic model of gas flow in barred galaxies is consistent with the kinematics of the molecular gas in this set of seven galaxies. The gas quickly changes velocity upon entering the dust lanes along the leading edge of the bar to flow directly down the dust lane. At the contact point of the dust lane and the nuclear ring, when the projections are favorable, we see that there are two velocity components. One is the slower rotating component that is in near circular orbits around the nucleus. The other is the faster flowing gas that traveled down the dust lane and is now flowing back into the bar region. We have clearly detected this gas spraying back into the bar region in those galaxies with orientations favorable for detection.

We have also shown that a dense ISM consisting of ballistic clouds is not consistent with the observed kinematics in the dust lanes. The observed velocity line widths in the dust lanes are instead consistent with the dense ISM undergoing a shock similar to the one the diffuse ISM is experiencing in NGC 1530 (RVT97). These observations do not preclude the dense ISM from consisting of clouds. They only require that the clouds respond to the

hydrodynamic forces that operate on an ideal gas. 
The authors would like to thank John Lugten for the reduction of the NGC 5135 data. They would also like to acknowledge helpful discussions with Peter Teuben and Jim Stone. Support for this work was provided by NASA through Hubble Fellowship grant HF-01100.01 awarded by the Space Telescope Science Institute, which is operated by the Association of Universities for Research in Astronomy, Inc., for NASA under contract 5-26555. We would like to thank the referee for comments that improved the paper. 


\section{REFERENCES}

Athanassoula, E. 1992a, MNRAS, 259, 328

Athanassoula. E. 1992b, MNRAS, 259, 345

Barton, I. J. \& Thompson, L. A. 1997, AJ, 114, 655

Benedict, G. F. et al. 1993, AJ, 105, 1369

Benedict, G. F., Smith, B. J., \& Kenney, J. D. P. 1996, AJ, 111,1861

Burbidge, E. M., Burbidge, G. R., \& Prenderdast, K. H. 1962, ApJ, 136, 704

Buta, R. 1986, ApJS, 61, 631

Buta, R. \& Purcell, G. B. 1998, AJ, 115, 484

Byrd, G., Rautiainen, P., Salo, H., Buta, R., \& Crocher, D.A. 1994, AJ, 108, 476

Combes, F. 1996, in IAU Colloqium 157, Barred Galaxies, eds. R. Buta, D. A. Crocker, \& B.G. Elmegreen, (San Francisco:ASP), 286

Combes, F., \& Gerin, M. 1985, A\&A, 150, 327

de Vaucouleurs, G., de Vaucouleurs, A., Corwin, H. G., Jr., Buta, R. J., Paturel, G., \& Foque, P. 1991, Third Reference Catalogue of Bright Glaxies (New York: Springer)(RC3)

Downes, D., Reynaud, D., Solomon, P. M., \& Radford, S. J. E. 1996, ApJ, 461, 186

Duval, M. F. \& Athanassoula, E. 1983, A\&A, 121, 297

Elmegreen, B. G. \& Elmegreen, D. M. 1985, ApJ, 288, 438

Elmegreen, D. M., Elmegreen, B. G., Chromey, F. R., \& Hasselbacher, D. A. 1996, ApJ, 469, 131

England, M. N. 1989, ApJ, 344, 669

England, M. N., Gottesman, S. T., \& Hunter, J. H., Jr. 1990, ApJ, 348, 456

Friedli, D. \& Benz, W. 1993, A\&A, 268, 65

Handa, T., Nakai, N., Sofue, Y., Hayashi, M., \& Fujimoto, M. 1990, PASJ, 42, 1

Haynes, M. P., Giovanelli, R., \& Roberts, M. S. 1979, ApJ, 229, 83

Heckman, T. M., Blitz, L., Wilson, A. S., Armus, L., \& Miley, G. K. 1989, ApJ, 342, 735

Heller, C. H., \& Shlosman, I. 1994, ApJ, 424, 84

Huntley, J. M. 1978, ApJ, 225, 101 
Jackson, J. M., Eckart, A., Cameron, M., Wild, W., Ho, P. T. P., Pogge, R. W., \& Harris, A. I. 1991, ApJ, 375, 105

Jorsater, S., \& van Moorsel, G. A. 1995, AJ, 110, 2037

Kenney, J. D. P. \& Lord, S. D. 1991, ApJ, 381, 118

Kenney, J. D. P., Wilson, C. D., Scoville, N. Z., Devereux, N. A., \& Young, J. S. 1992, ApJ, 395, L79

Lindblad, P. A. B., Kristen, H., Jorsater, S., \& Hogbom, J. 1997, A\&A, 317, 36

Merrifield, M. R. \& Kuijken, K. 1995, MNRAS, 274, 933

Mulchaey, J. S., Regan, M. W., \& Kundu, A. 1997, ApJS, 110, 299

Norman, C. A., Sellwood, J. A., \& Hasan, H. 1996, ApJ, 462, 114

Palous, J., Jungwiert, B., \& Kopecky, J. 1993, A\&A, 274, 189

Peterson, C. J., Thonnard, N., Rubin, V. C., \& Ford, W. K., Jr. 1978, ApJ, 219, 31

Phillips, M. M., Charles, P. A., Baldwin, J. A. 1983, ApJ, 266, 485

Piner, B. G., Stone, J. M., \& Teuben, P. J. 1995, ApJ, 449, 508

Planesas, P., Colina, L., \& Perez-Olea, D. 1997, A\&A, 325, 81

Pogge, R. W. 1989, ApJS, 71, 433

Rice, W., Lonsdale, C. J., Soifer, B. T., Neugebauer, G., Koplan, E. L., Lloyd, L. A., De Jong, T., \& Habing, H. J. 1988, ApJS, 68, 91

Regan, M. W. \& Elmegreen, D. M. 1997, AJ, 114, 965

Regan, M. W., Vogel, S. N., \& Teuben, P. J. 1995, ApJ, 449, 576

Regan, M. W., Vogel, S. N., \& Teuben, P. J. 1997, ApJ, 482, L143

Regan, M. W., Vogel, S. N., Teuben, P. J., vad der Hulst, T. 1996, AJ, 112, 2549

Reuter, H.-P., Krause, M., Wielebinski, R. Lesch, H. 1991, A\&A, 248, 12

Reuter, H.-P. Siefers, A. W., Pohl, M., Lesch, H., Wielebinski, R. 1996, A\&A, 306, 721

Reynaud, D. \& Downes, D. 1997, A\&A, 319, 737

Reynaud, D. \& Downes, D. 1998, A\&A, in press

Roberts, W. W. Jr \& Hausman, M. A. 1984, ApJ, 277, 744

Rots, A. H. 1978, AJ, 83, 219

Ryder, S., Buta, R., \& Toledo, H. 1996 in IAU Colloqium 157, Barred Galaxies, eds. R. Buta, D. A. Crocker, \& B.G. Elmegreen, (San Francisco:ASP), 157 
Sancisi, R., Allen, R. J., \& Sullivan W. T., III 1979, A\&A, 78,217

Sandage, A. 1961, The Hubble Atlas of Galaxies (Publ. 618; Washington, Carnegie Institution of Washington)

Sanders, R. H. \& Tubbs, A. D. 1980, ApJ, 235, 803

Sault, R. J., Staveley-Smith, L., Brouw, W. N. 1996, A\&AS, 120, 375

Schwarz, M. P. 1984, MNRAS, 209, 93

Sheth, K., Regan, M. W., \& Vogel, S. N. 1998, ApJ, submitted

Shlosman, I., Frank, J., \& Begelman, M. C. 1989, Nature, 338, 45

Smith, B. J., Harvey, P. M., Colome, C., Zhang, C. Y., Difrancesco, J., \& Pogge, R. W. 1994, ApJ, 425, 91

Teuben, P. J., Sanders, R. H., Atherton, P. D., \& van Albada, G. D. 1986, MNRAS, 221, 1

Thuan, T. X. 1984, ApJ, 281, 126

Tubbs, A. D. 1982, ApJ, 255, 458

Wynn-Williams, C. G. \& Becklin, E. E. 1985, ApJ, 290, 108

Young, J. S., Xie, S., Kenney, J. D. P., \& Rice, W.L. 1989, ApJ, 70,699

Zhang, X., Wright, M., \& Alexander, P. 1993, ApJ, 418, 100 
Fig. 1.- NGC 1300 a) CO total intensity (contours) overlaid on a near infrared image K-band (gray-scale) from Regan and Elmegreen (1997). The grey contours are the intensity from the receding side of the galaxy and the white contours are from the approaching side of the galaxy. The dotted line circle shows the half-power radius of the primary beam, and the synthesized beam is shown in the lower right. b) CO total intensity for the central region of NGC 1300. The bottom left of each letter marks the locations at which the spectra presented in c) were derived. The region displayed is outlined with a rectangle in part a). The contours are at 2, 4, 6, and 8 times the noise in the map which is $4.3 \mathrm{Jy} \mathrm{km} \mathrm{s}$. The solid line is the major axis of the galaxy with the thicker half being the receding side and the thin line the approaching side. The dashed line represents the minor axis with the thicker line being the far side. c) Spectra of CO emission from NGC 1300 derived from the bottom left corner of the letters plotted in b). The systemic velocity is represented by the dotted line. The resolution is $10 \mathrm{~km}^{-1}$, and the noise in each channel is $88 \mathrm{mJy}$.

Fig. 2.- Same as Figure 1 except where noted. NGC 1530 a) CO total intensity (contours) overlaid on a near infrared image K-band (gray-scale) from Regan et al. (1995). b) CO total intensity for the central region of NGC 1530 with the locations of the spectra marked. The contours are at 2, 4, 6, and 8 times the noise in the map which is $4.3 \mathrm{Jy} \mathrm{km} \mathrm{s}^{-1}$. c) Spectra of CO emission from NGC 1530 at the locations marked in b). The noise in each channel is $49 \mathrm{mJy}$.

Fig. 3.- Same as Figure 1 except where noted. NGC 2903 a) CO total intensity (contours) overlaid on a near infrared image K-band (gray-scale) from Regan and Elmegreen (1997). b) CO total intensity for the central region of NGC 2903 with the locations of the spectra marked. The contours are at 2, 4, 6, and 8 times the noise in the map which is $2.5 \mathrm{Jy} \mathrm{km}$ $\mathrm{s}^{-1}$. c) Spectra of CO emission from NGC 2903 at the locations marked in b). The noise in each channel is $48 \mathrm{mJy}$.

Fig. 4.- Same as Figure 1 except where noted. NGC 3627 a) CO total intensity (contours) overlaid on a near infrared image K-band (gray-scale) from Regan and Elmegreen (1997). The dotted line circles show the half-power points of the primary beams of each of the pointings for the mosaic. b) CO total intensity for the central region of NGC 3627 with the locations of the spectra marked. The contours are at 2, 4, 6, 10, 15, 20, 25, 30, 35, 40, 45, $50,55,60,65,70$ times the noise in the map, $3.2 \mathrm{Jy} \mathrm{km} \mathrm{s}^{-1}$. c) Spectra of CO emission from NGC 3627 at the locations marked in b). The noise in each channel is $50 \mathrm{mJy}$. 
Fig. 5.- Same as Figure 1 except where noted. NGC 4314 a) CO total intensity (contours) overlaid on a near infrared image K-band (gray-scale) from Regan and Elmegreen (1997). b) CO total intensity for the central region of NGC 4314 with the locations of the spectra marked. The contours are at 2, 4, 6, and 8 times the noise in the map which is $1.8 \mathrm{Jy} \mathrm{km}$ $\mathrm{s}^{-1}$. c) Spectra of CO emission from NGC 4314 at the locations marked in b). The noise in each channel is $38 \mathrm{mJy}$.

Fig. 6. - Same as Figure 1 except where noted. NGC 5135 a) CO total intensity (contours) overlaid on a near infrared image K-band (gray-scale) from Mulchaey, Regan, \& Kundu (1997). b) CO total intensity for the central region of NGC 5135 with the locations of the spectra marked. The contours are at 2, 4, 6, and 8 times the noise in the map which is 3.8 Jy $\mathrm{km} \mathrm{s}^{-1}$. c) Spectra of CO emission from NGC 5135 at the locations marked in b). The noise in each channel is $84 \mathrm{mJy}$.

Fig. 7.- Same as Figure 1 except where noted. NGC 5383 a) CO total intensity (contours) overlaid on a near infrared image K-band (gray-scale) from Regan and Elmegreen (1997). b) CO total intensity for the central region of NGC 5383 with the locations of the spectra marked. The contours are at 2, 4, 6, and 8 times the noise in the map which is $3.3 \mathrm{Jy} \mathrm{km}$ $\mathrm{s}^{-1}$. c) Spectra of CO emission from NGC 5383 at the locations marked in b). The noise in each channel is $54 \mathrm{mJy}$.

Fig. 8. - Gas density from hydrodynamic model. The three regions of interest, the dust lane, the contact point of the dust lane and the nuclear ring, and the spray region are marked.

Fig. 9.- Gas velocity from the hydrodynamic model in the rotating reference frame of the bar. The background gray-scale is the gas surface density. The arrow represents the direction and magnitude of the gas velocity. Notice how the gas makes an abrupt change in direction when it enters and flows down the dust lane. At the contact point all of the gas is flowing tangentially and it is clear that the gas in the ring (white arrow) is moving slower than the gas just outside of the ring. In the spray region the gas flow is diverging from the contact point.

Fig. 10.- Orbits of gas clouds in a barred potential under the assumption that the ISM is composed of ballistic clouds that interact only through collisions. The bar major axis is shown by the vertical line. The bar is rotating counter-clockwise. The thick circle represents the nuclear ring. 
Table 1. Galaxy Characteristics

\begin{tabular}{|c|c|c|c|c|c|}
\hline Galaxy & $\begin{array}{c}\text { R.A. } \\
\text { J2000 }\end{array}$ & $\begin{array}{c}\text { Dec. } \\
\text { J2000 }\end{array}$ & $\begin{array}{l}\text { Hubble } \\
\text { Type }\end{array}$ & $\begin{array}{l}\text { Systemic } \\
\text { Velocity }^{\mathrm{a}}\end{array}$ & Linear Scale ${ }^{b}$ \\
\hline NGC 1300 & $3^{h} 19^{m} 41^{\mathrm{s}} .6$ & $-19^{\circ} 24^{\prime} 43^{\prime \prime} .1$ & $\left(\mathrm{R}^{\prime}\right) \mathrm{SB}(\mathrm{s}) \mathrm{bc}$ & $1552 \mathrm{~km} \mathrm{~s}^{-1}$ & $92 \mathrm{pc} \operatorname{arcsec}^{-1}$ \\
\hline NGC 1530 & $4^{h} 23^{m} 26^{\mathrm{s}} .7$ & $75^{\circ} 17^{\prime} 43^{\prime \prime} .8$ & $\mathrm{SB}(\mathrm{rs}) \mathrm{b}$ & $2467 \mathrm{~km} \mathrm{~s}^{-1}$ & $157 \mathrm{pc} \operatorname{arcsec}^{-1}$ \\
\hline NGC 2903 & $9^{h} 32^{m} 10^{s} .1$ & $21^{\circ} 30^{\prime} 02^{\prime \prime} .0$ & $\mathrm{SAB}(\mathrm{rs}) \mathrm{bc}$ & $550 \mathrm{~km} \mathrm{~s}^{-1}$ & $55 \mathrm{pc}_{\operatorname{arcsec}}^{-1}$ \\
\hline NGC 3627 & $11^{h} 20^{m} 15^{\mathrm{s}} .1$ & $12^{\circ} 59^{\prime} 21^{\prime \prime} .7$ & $\mathrm{SAB}(\mathrm{s}) \mathrm{b}$ & $726 \mathrm{~km} \mathrm{~s}^{-1}$ & $70 \mathrm{pc} \operatorname{arcsec}^{-1}$ \\
\hline NGC 4314 & $12^{h} 22^{m} 32^{\mathrm{s}} .0$ & $29^{\circ} 53^{\prime} 43^{\prime \prime} \cdot 3$ & $\mathrm{SB}(\mathrm{rs}) \mathrm{a}$ & $969 \mathrm{~km} \mathrm{~s}^{-1}$ & $81 \mathrm{pc} \operatorname{arcsec}^{-1}$ \\
\hline NGC 5135 & $13^{h} 25^{m} 44^{\mathrm{s}} .0$ & $-29^{\circ} 50^{\prime} 02^{\prime \prime} .3$ & $\mathrm{SB}(\mathrm{l}) \mathrm{ab}$ & $4112 \mathrm{~km} \mathrm{~s}^{-1}$ & $286 \mathrm{pc} \operatorname{arcsec}^{-1}$ \\
\hline NGC 5383 & $13^{h} 57^{m} 04^{\mathrm{s}} .6$ & $41^{\circ} 50^{\prime} 46^{\prime \prime} .0$ & $\left(\mathrm{R}^{\prime}\right) \mathrm{SB}(\mathrm{rs}) \mathrm{b}$ pec & $2263 \mathrm{~km} \mathrm{~s}^{-1}$ & 159 pc $\operatorname{arcsec}^{-1}$ \\
\hline
\end{tabular}

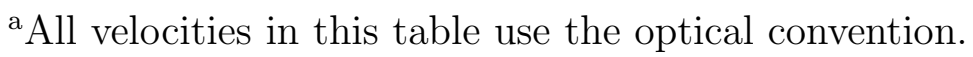

b The linear scale was determined by using $\mathrm{H}_{0}=75 \mathrm{~km} \mathrm{~s}^{-1} \mathrm{Mpc}^{-1}$ and the $\mathrm{V}_{3 k}$ from the RC3.

Table 2. CO Observational Parameters

\begin{tabular}{|c|c|c|c|c|c|}
\hline Galaxy & $\begin{array}{c}\text { Bandwidth } \\
\text { (Total, Imaged) }\end{array}$ & $\begin{array}{l}\text { Projected } \\
\text { Baselines }\end{array}$ & $\mathrm{T}_{\text {sys }}$ & $\begin{array}{l}\text { \# of Config. x } \\
\text { \# of Antennas }\end{array}$ & $\theta_{\text {synth }}$ \\
\hline NGC 1300 & $912,400 \mathrm{~km} \mathrm{~s}^{-1}$ & $2.2-50 \mathrm{k} \lambda$ & 700-1500 K & $1 \mathrm{x} 9$ & $8.7 \times 3.8$ \\
\hline NGC 1530 & $772,490 \mathrm{~km} \mathrm{~s}^{-1}$ & $2.8-90 \mathrm{k} \lambda$ & 500-1000 K & $3 \times 6$ & $5^{\prime \prime} .1 \times 4^{\prime \prime} .3$ \\
\hline NGC 2903 & $516,400 \mathrm{~km} \mathrm{~s}^{-1}$ & $2.4-83 \mathrm{k} \lambda$ & $400-800 \mathrm{~K}$ & $3 \times 6$ & $3.9 \times 3.5$ \\
\hline NGC 3627 & $900,460 \mathrm{~km} \mathrm{~s}^{-1}$ & $2.2-27 \mathrm{k} \lambda$ & $300-600 \mathrm{~K}$ & $1 \mathrm{x} 9$ & $7^{\prime \prime} .7 \times 7^{\prime \prime} ! 1$ \\
\hline NGC 4314 & $516,420 \mathrm{~km} \mathrm{~s}^{-1}$ & $2.2-83 \mathrm{k} \lambda$ & $400-700 \mathrm{~K}$ & $3 \times 6$ & $5^{\prime \prime} .6 \times 4^{\prime \prime} .2$ \\
\hline NGC 5135 & $730,400 \mathrm{~km} \mathrm{~s}^{-1}$ & $1.4-75 \mathrm{k} \lambda$ & $800-1500 \mathrm{~K}$ & $4 \times 6$ & $10^{\prime \prime} .2 \times 33^{\prime \prime} 6$ \\
\hline NGC 5383 & $460,460 \mathrm{~km} \mathrm{~s}^{-1}$ & $2.2-91 \mathrm{k} \lambda$ & $500-1100 \mathrm{~K}$ & $1 \times 9,3 \times 6$ & $4^{\prime \prime} .6 \times 4^{\prime \prime} .2$ \\
\hline
\end{tabular}


Table 3. Galaxy Position Angles

\begin{tabular}{crr}
\hline \hline Galaxy & Position Angle & Reference \\
\hline NGC 1300 & $-93 \pm 0.5^{\circ}$ & 1 \\
NGC 1530 & $8 \pm 1^{\circ}$ & 2 \\
NGC 2903 & $13 \pm 1^{\circ}$ & 3 \\
NGC 3627 & $-4 \pm 2^{\circ}$ & 3 \\
NGC 4314 & $-25 \pm 10^{\circ}$ & 4 \\
NGC 5135 & $-56 \pm 2^{\circ}$ & 3 \\
NGC 5383 & $85^{\circ}$ & 5 \\
& & \\
\hline
\end{tabular}

References. - (1) Lindblad et al. 1997

(2) Regan et al. 1996

(3) This Paper

(4) Benedict et al. 1996

(5) Duval \& Athanassoula 1983 

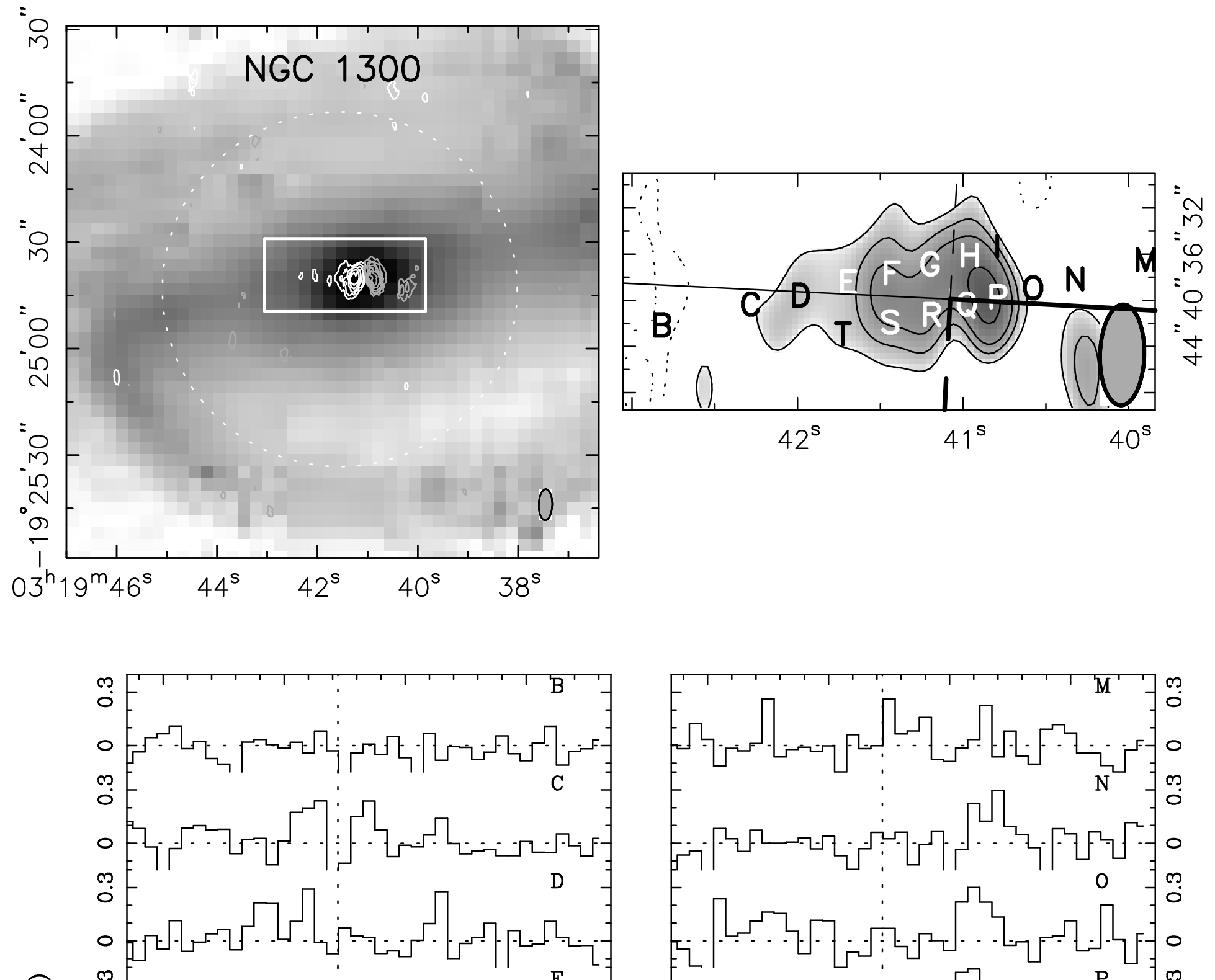

党:

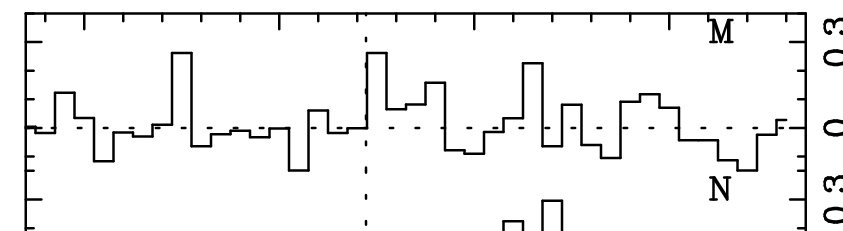

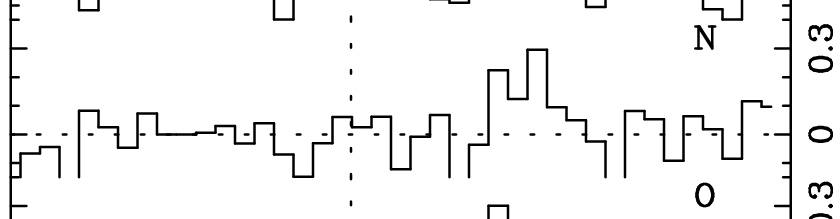
$=$

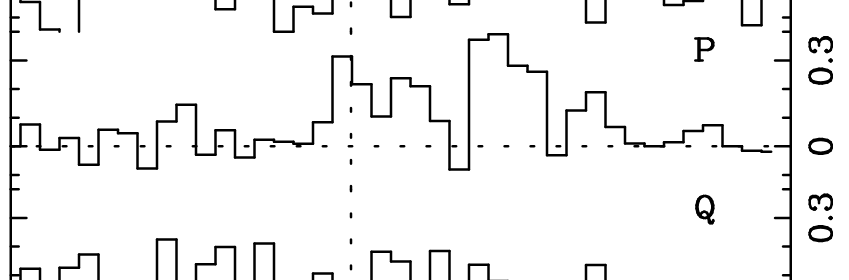

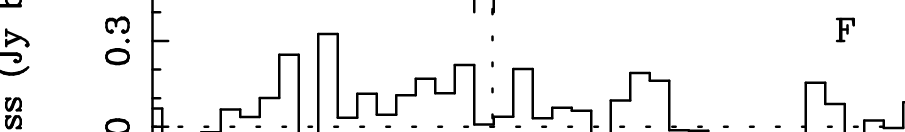

章

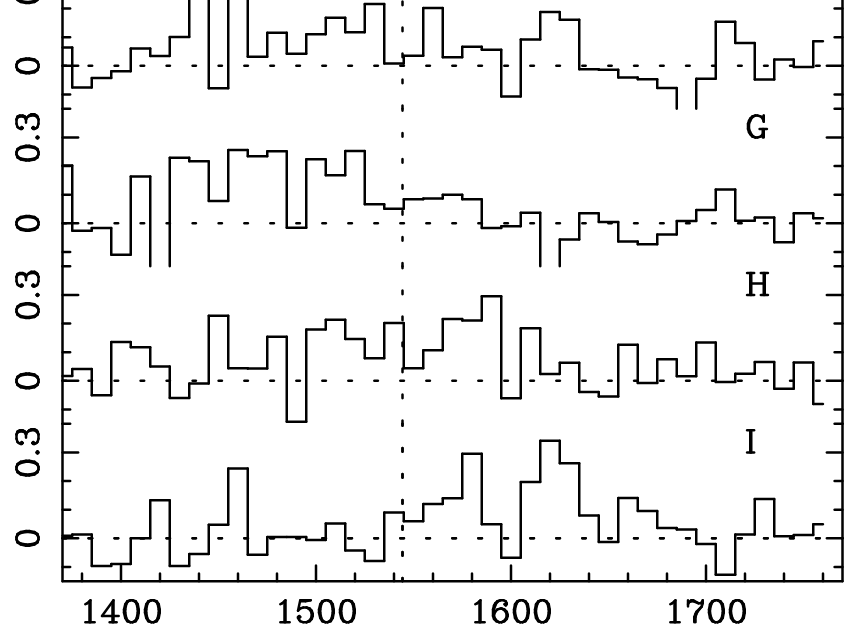

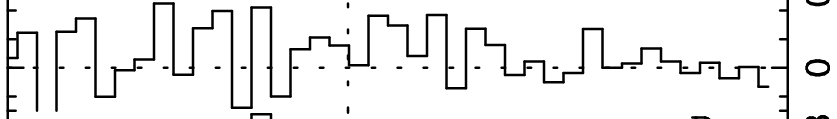
- च7 $\mathrm{R}$ -

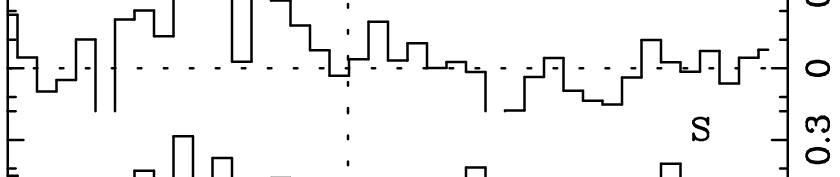

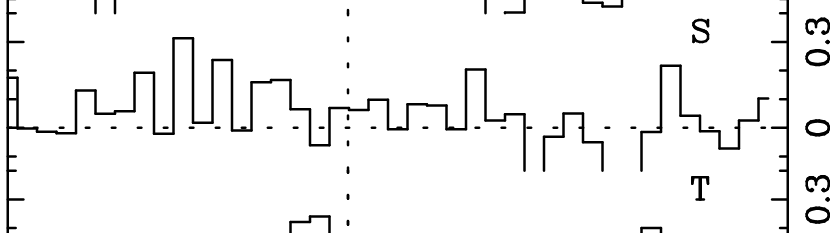
$\frac{1600}{1400} 1500$ Velocity $\left(\mathrm{km} \mathrm{s}^{-1}\right)$ 


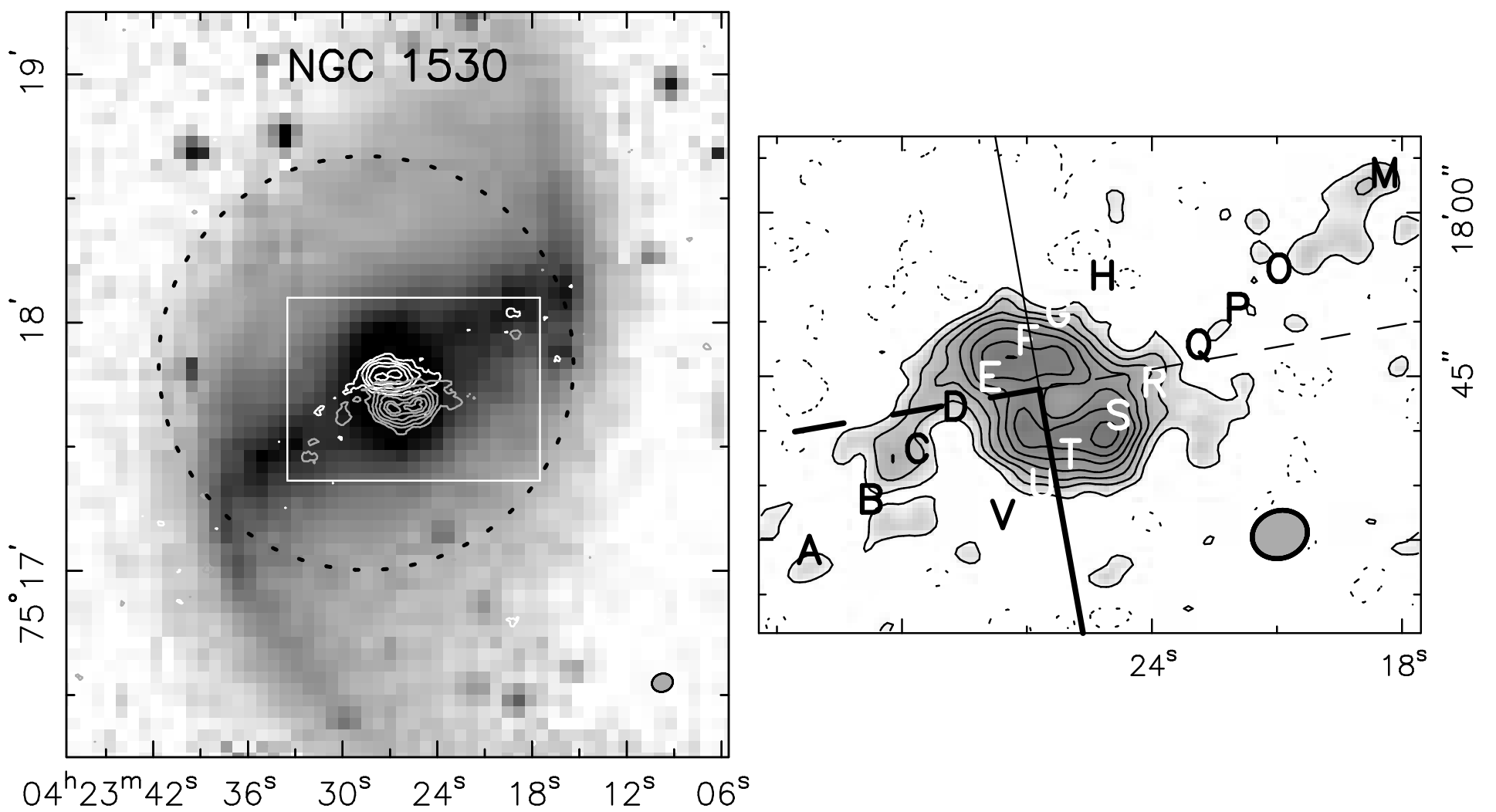

J2000

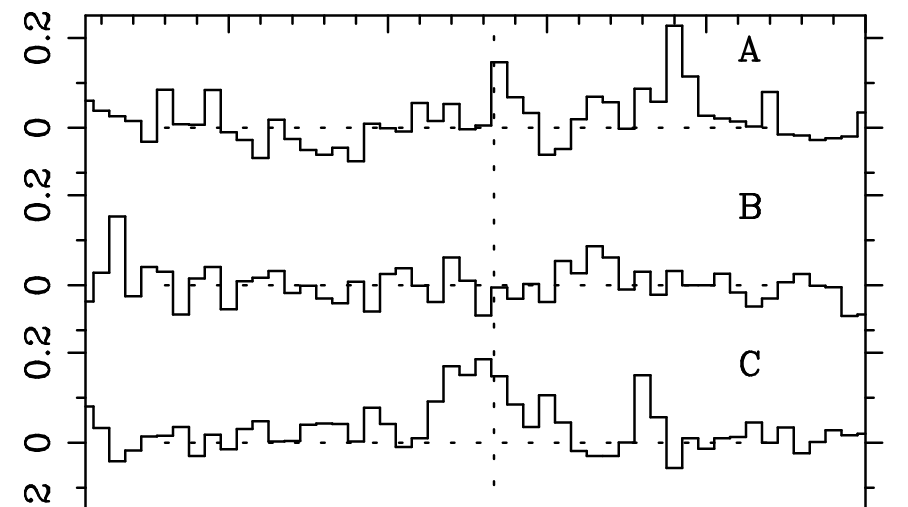

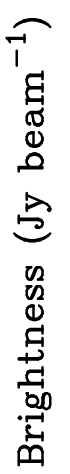

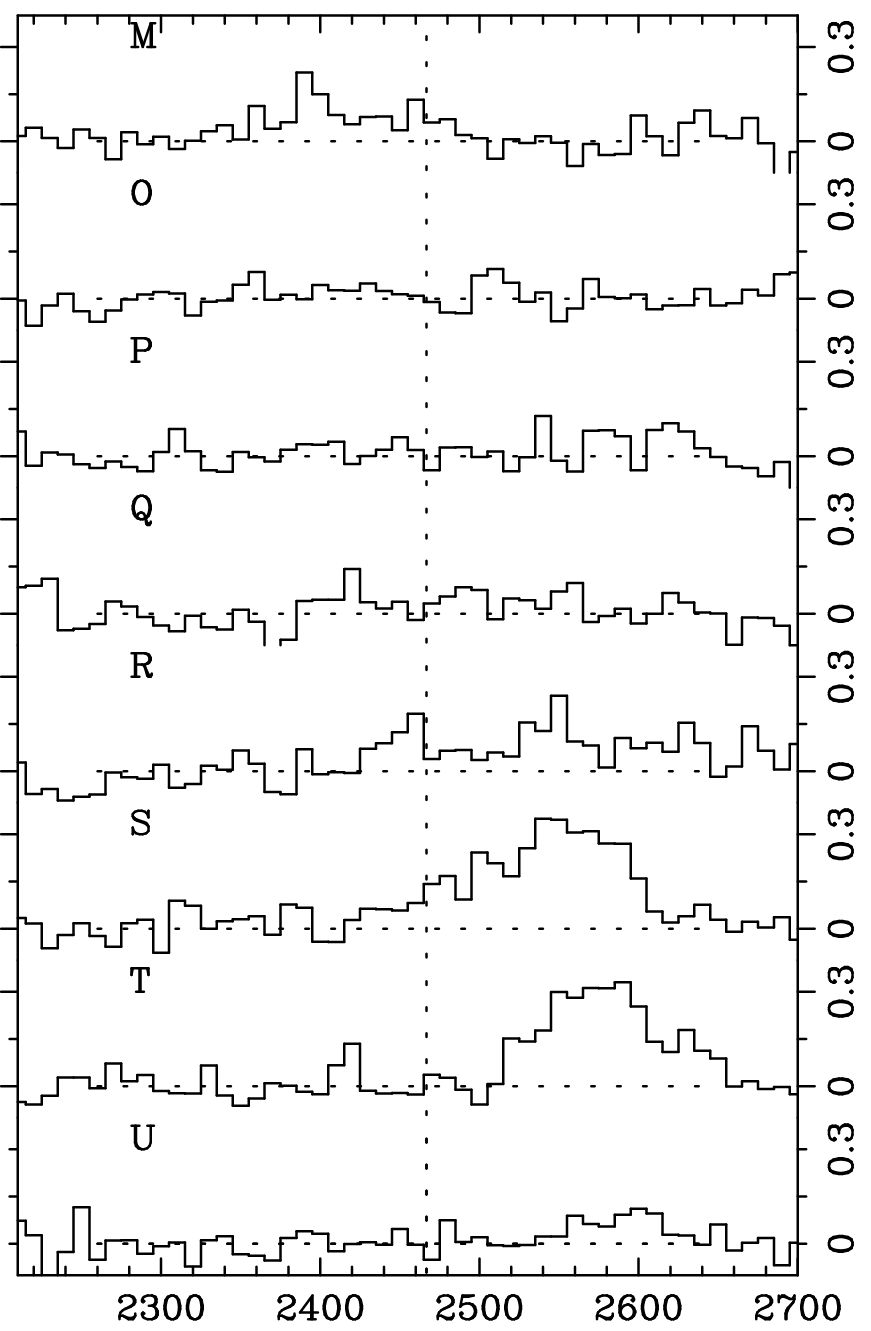

Velocity $\left(\mathrm{km} \mathrm{s}^{-1}\right)$ 

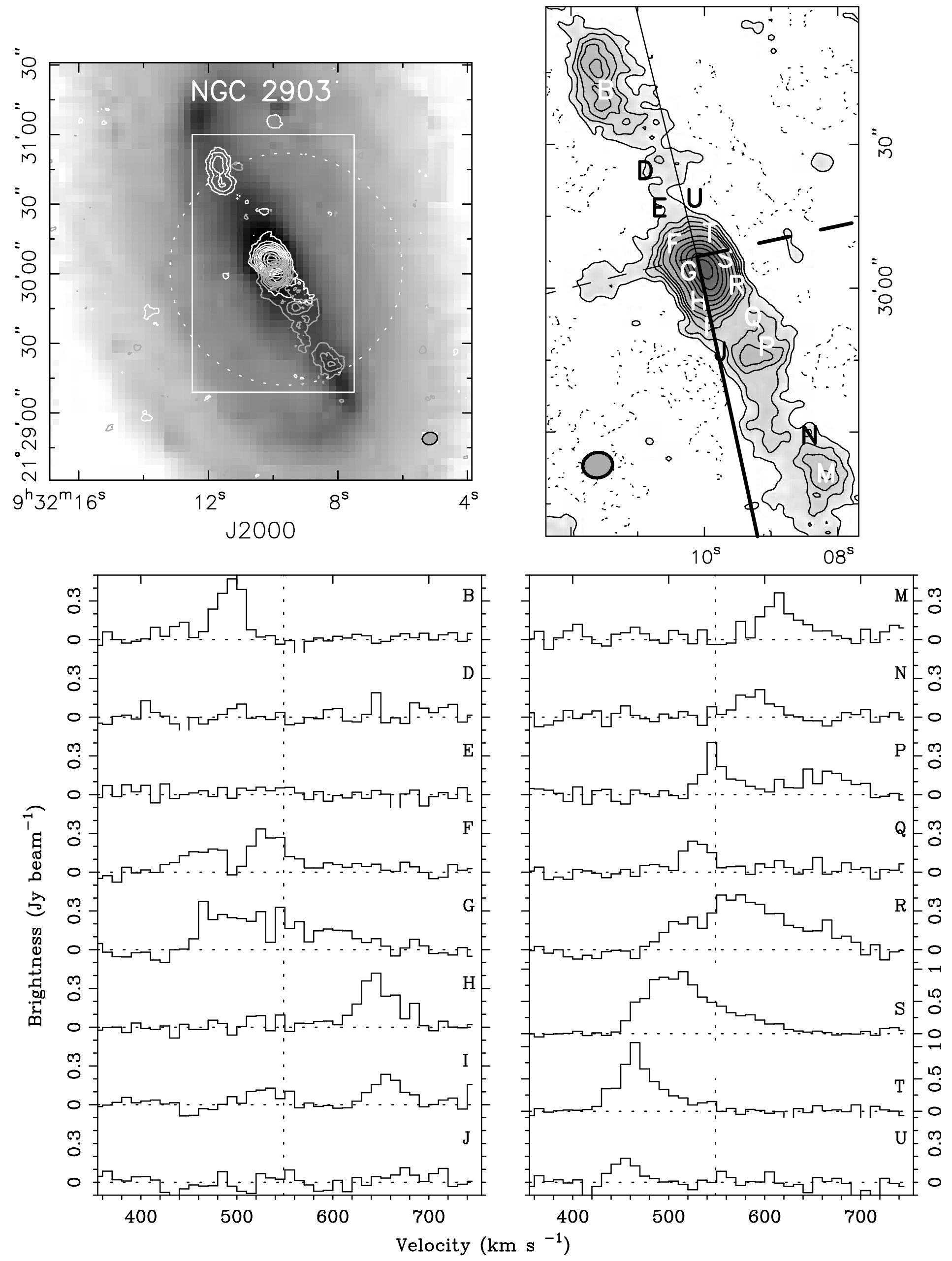


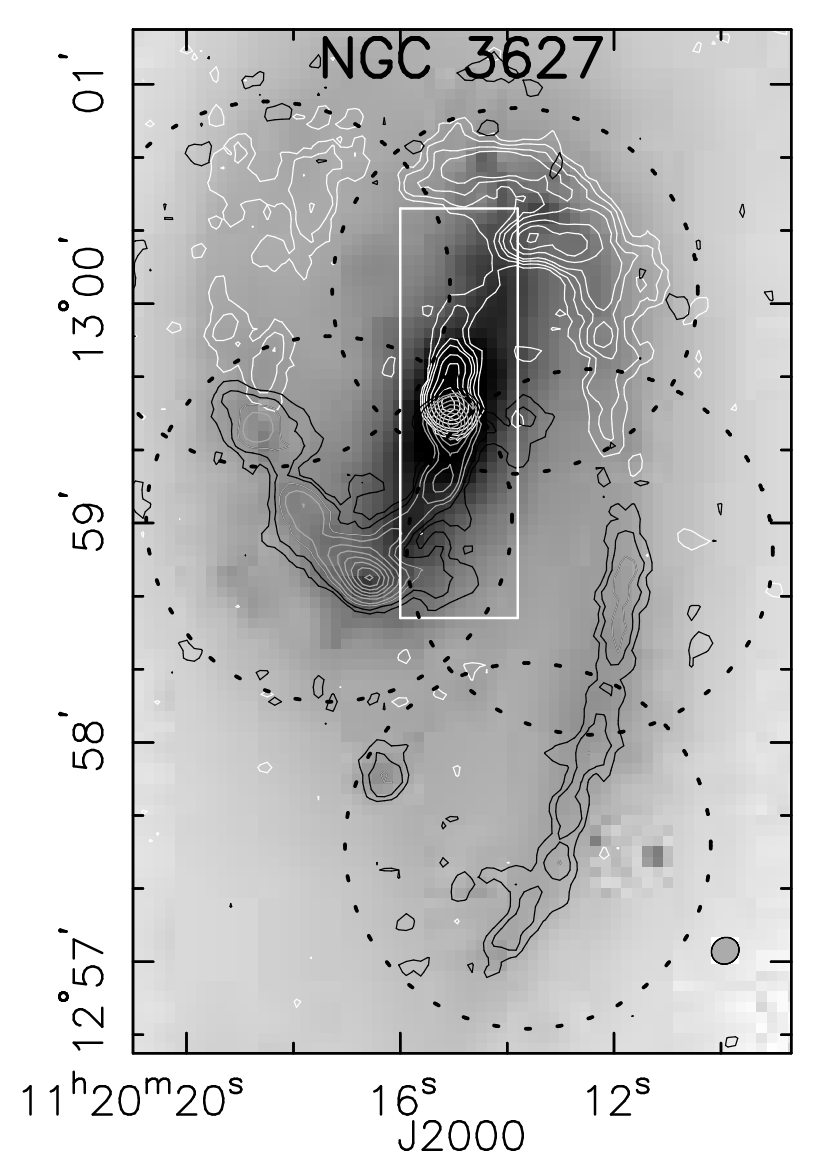

: 51 -

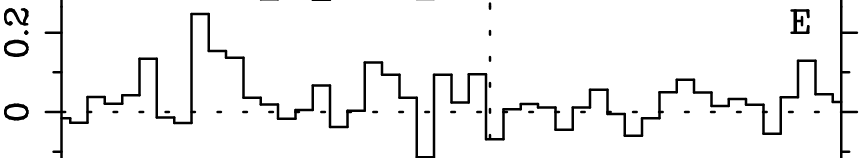
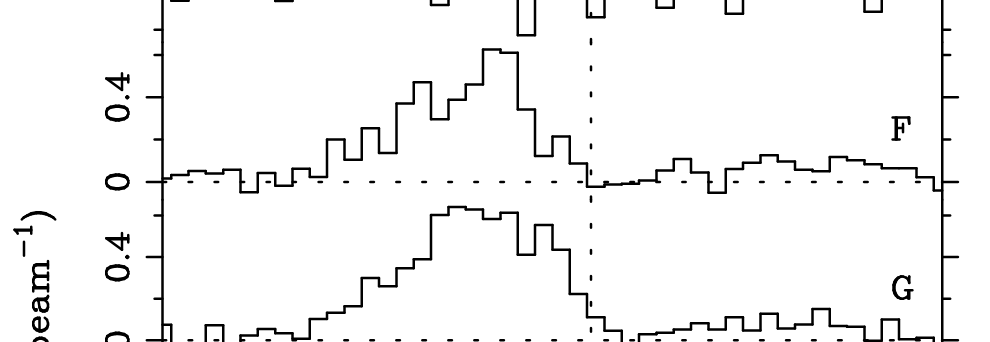

o ons

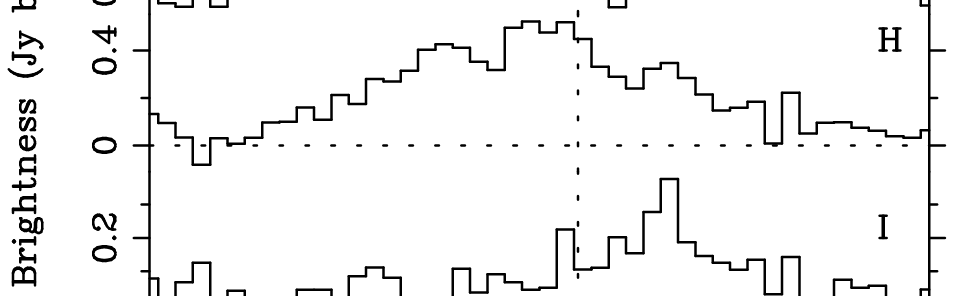

- Ir

$0 \frac{000}{000}$

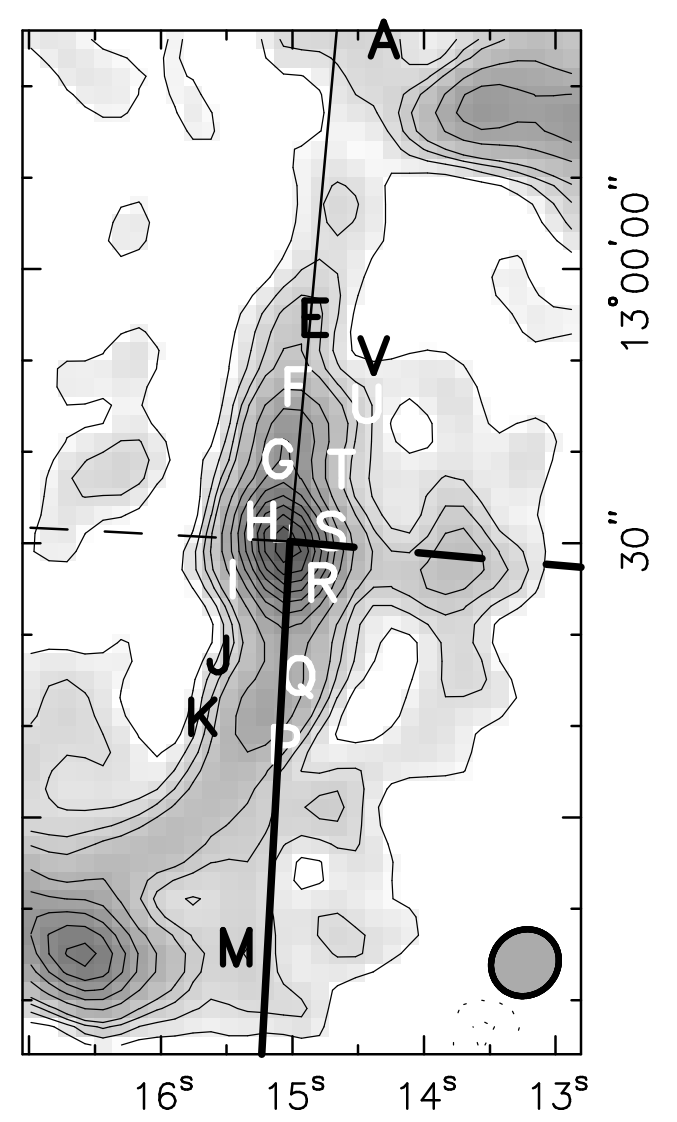

-

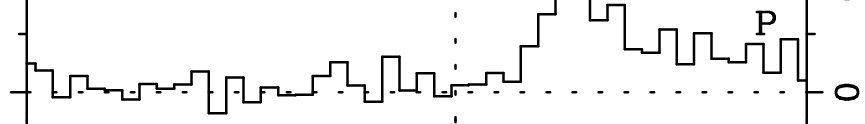

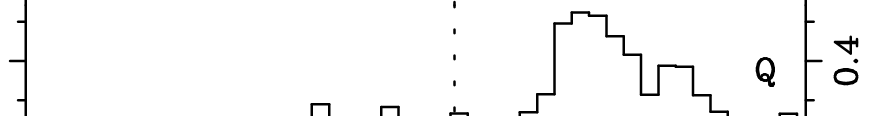

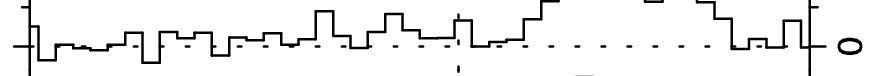

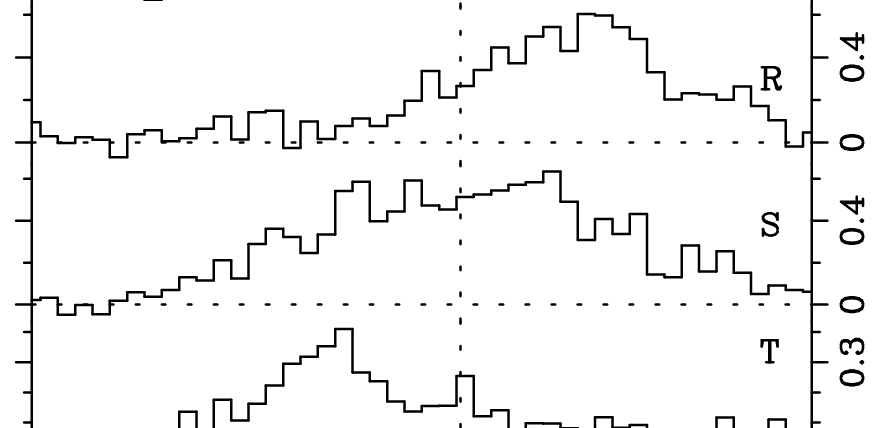
-

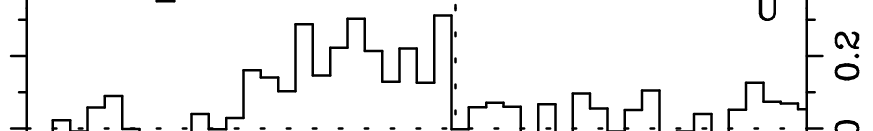
. $\underbrace{500}_{500}$

\section{Velocity $_{\text {LSR }}\left(\mathrm{km} \mathrm{s}^{-1}\right)$}



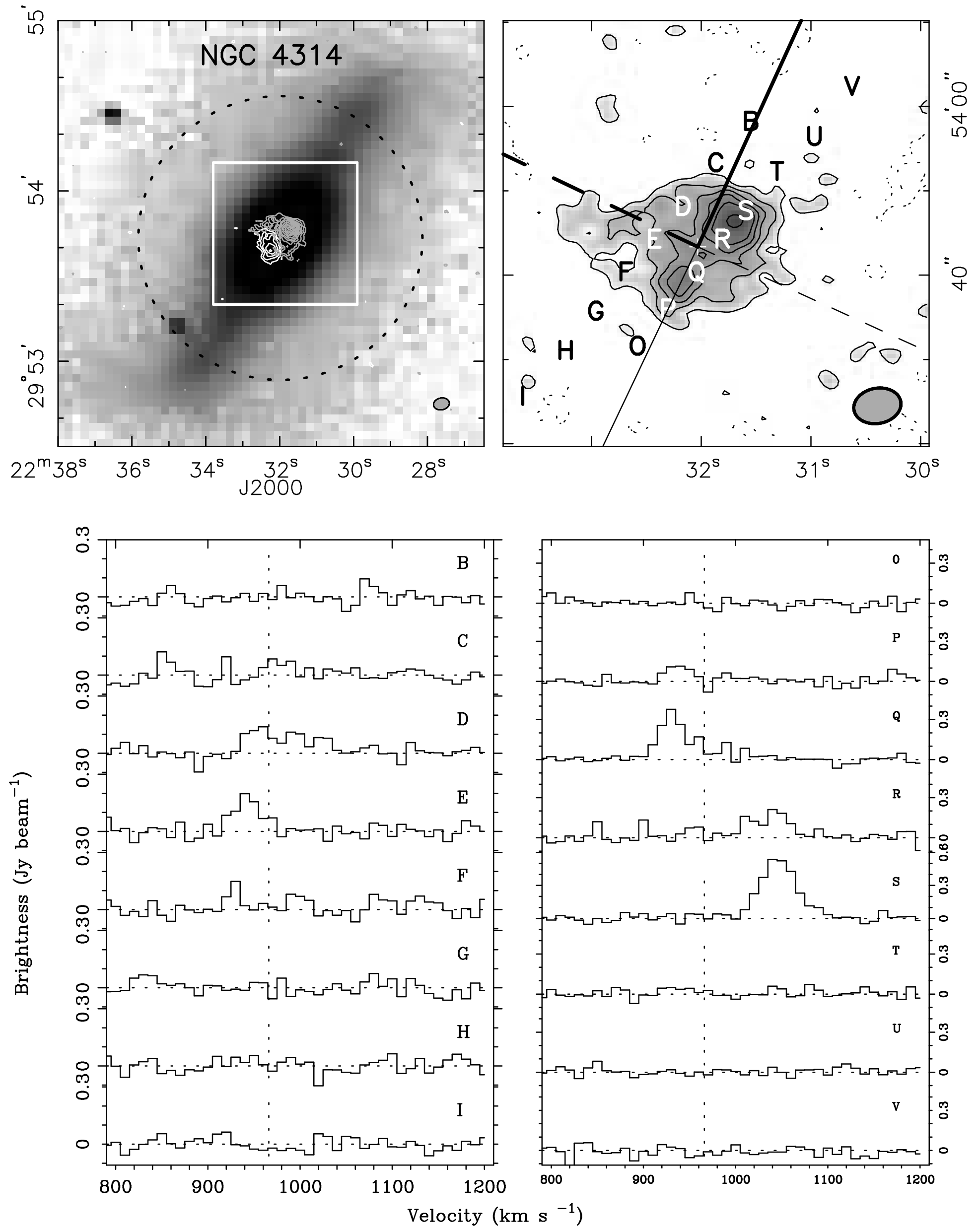

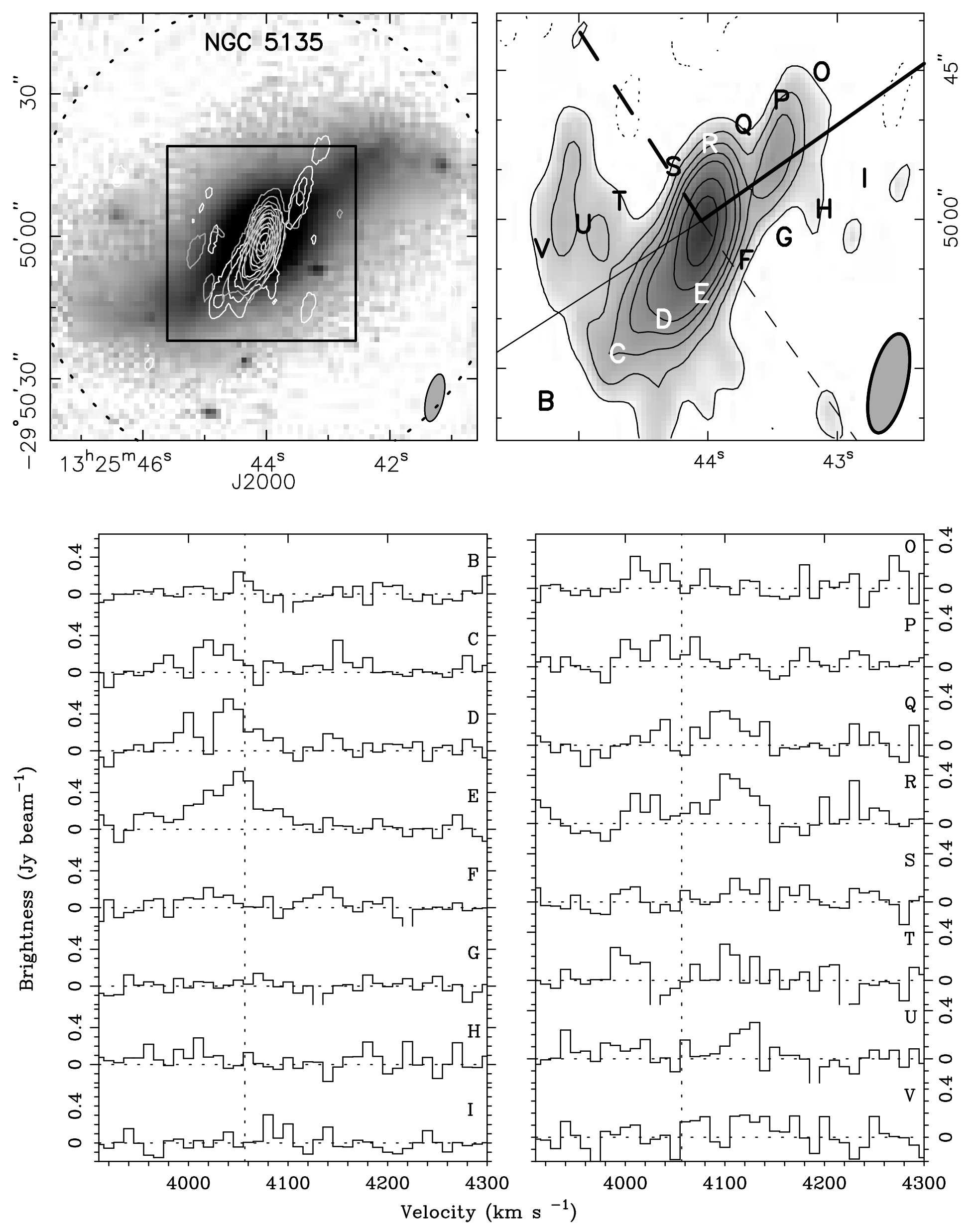

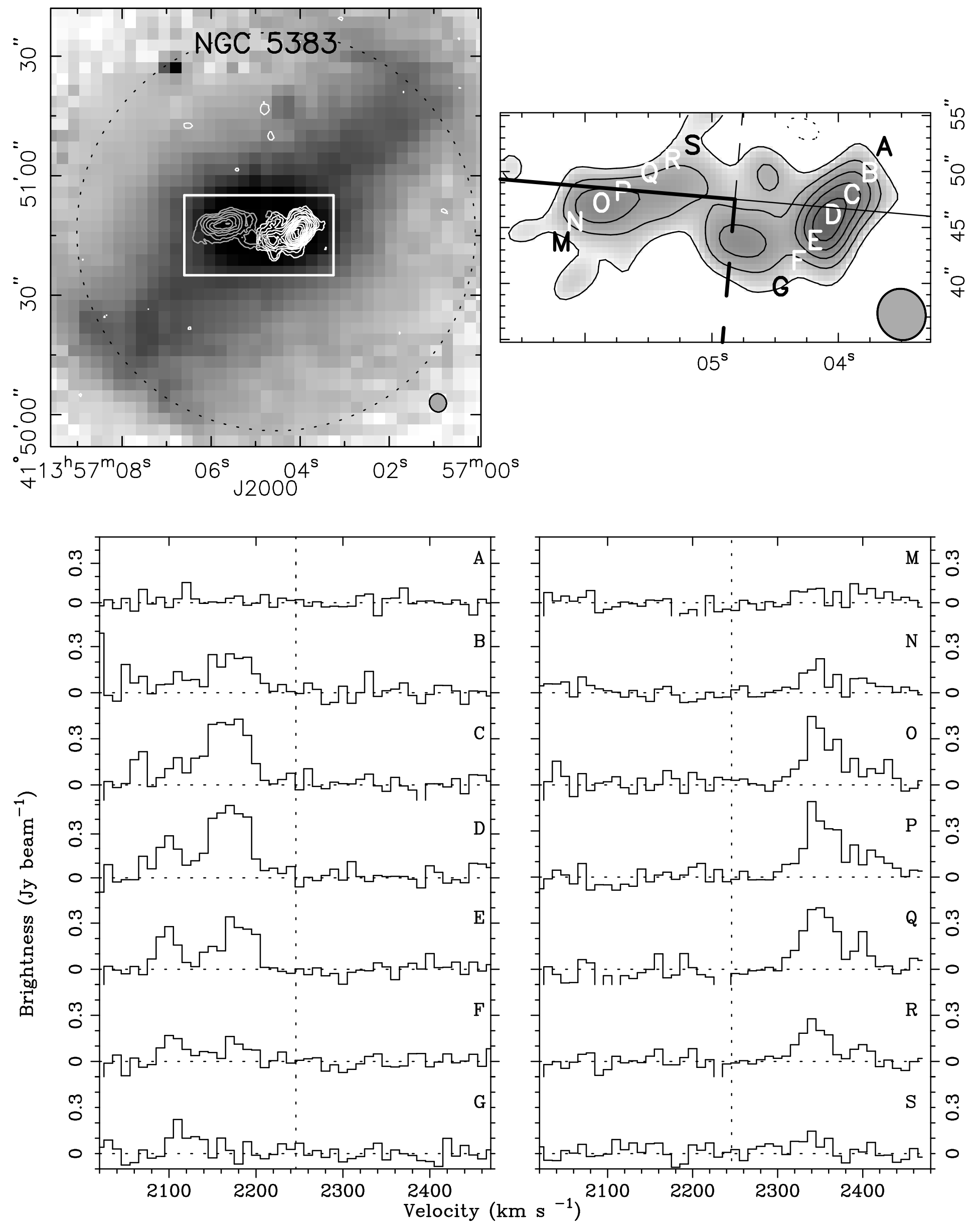


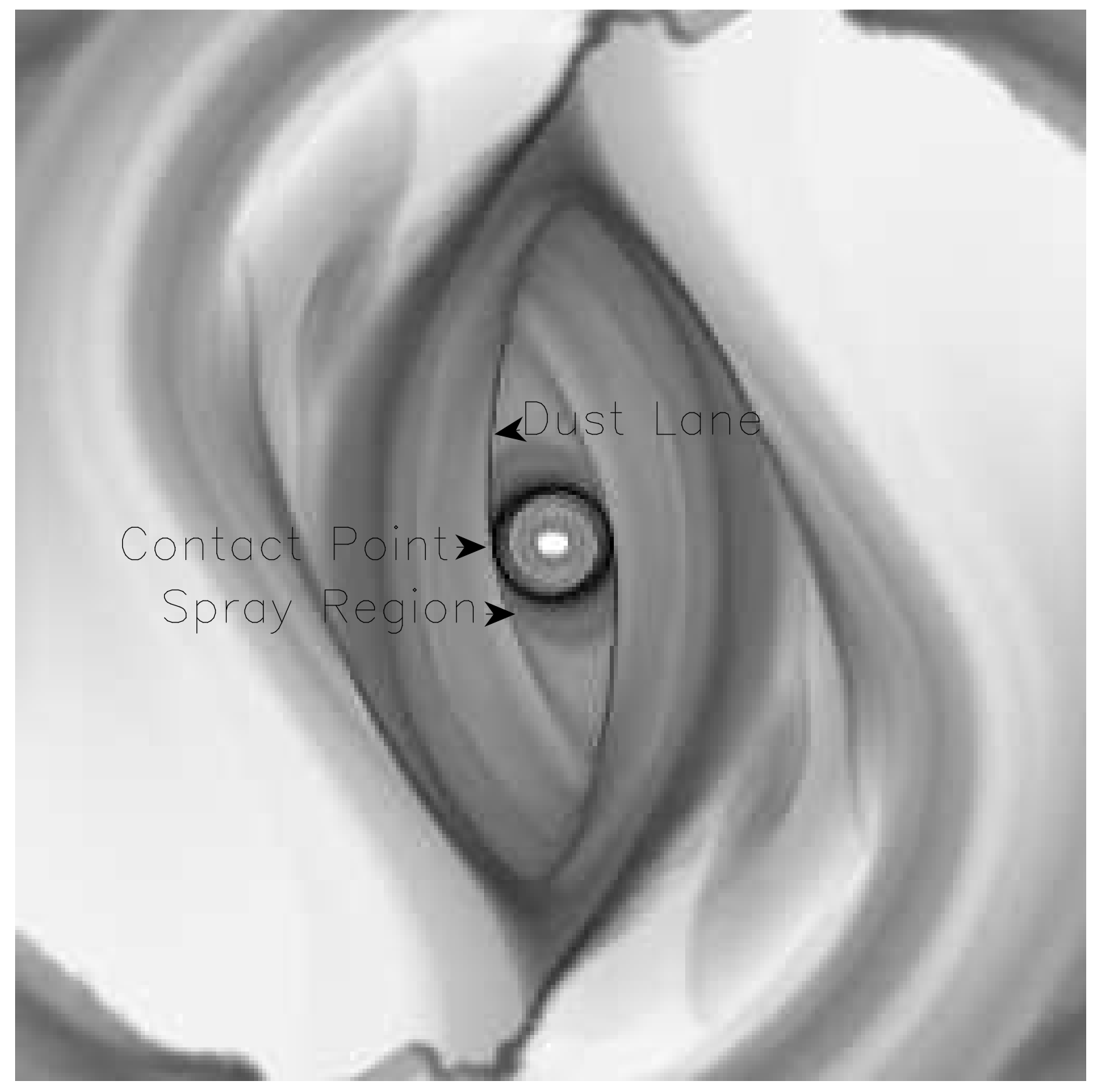




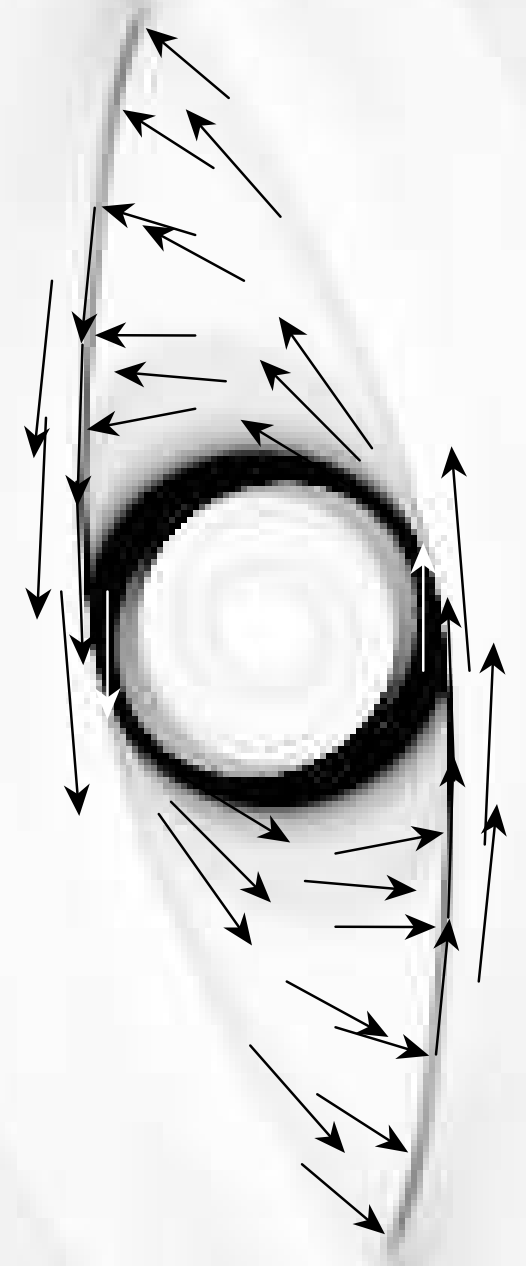


